\title{
Jan van Eyck's Perspectival System Elucidated Through Computer Vision ${ }^{1}$
}

Gilles Simon, Université de Lorraine, CNRS, Inria, LORIA, Nancy, France, gilles.simon@loria.fr

\begin{abstract}
It is generally accepted that Jan van Eyck was unaware of perspective. However, an a-contrario analysis of the vanishing points in five of his paintings, realized between 1432 and 1439, unveils a recurring fishbone-like pattern that could only emerge from the use of a polyscopic perspective machine with two degrees of freedom. A 3D reconstruction of Arnolfini Portrait compliant with this pattern suggests that van Eyck's device answered a both aesthetic and scientific questioning on how to represent space as closely as possible to human vision. This discovery makes van Eyck the father of today's immersive and nomadic creative media such as augmented reality and synthetic holography.
\end{abstract}

CCS CONCEPTS • Applied computing Arts and humanities

Additional Keywords: Jan Van Eyck, Perspective machine, Art history, Computer vision, Vanishing points.

\section{Introduction}

Perspective, as a physical model, describes the optical phenomenon at work in a camera obscura or dark chamber: Light enters the room through a small hole or lens, continues its path in the shape of a cone, then crosses a wall on which it deposits the image of its previous encounters. From this model laws were deduced, the first of which is described in the treatise De Pictura, written around 1435 by Leon Battista Alberti [1]: The orthogonals, or lines of the world perpendicular to the picture plane, meet, by perspective projection, at a single point, called the centric point by Alberti. This point corresponds to the orthogonal projection of the socalled view point (the projection center) onto the image plane.

\footnotetext{
${ }^{1}$ Permission to make digital or hard copies of all or part of this work for personal or classroom use is granted without fee provided that copies are not made or distributed for profit or commercial advantage and that copies bear this notice and the full citation on the first page.

Copyrights for components of this work owned by others than the author(s) must be honored. Abstracting with credit is permitted. To copy otherwise, or republish, to post on servers or to redistribute to lists, requires prior specific permission and/or a fee. Request permissions from Permissions@acm.org.
}

2577-6193/2021/08- 4:2 \$15.00

(C) Copyright is held by the owner/author(s). Publication rights licensed to ACM.

https://doi.org/10.1145/3465623 
In a biography written around 1465, Antonio Manetti attributes the first perspective representation to the Florentine architect Filippo Brunelleschi (1377-1446) [2]. Painted around 1415 on a wooden panel, it represented the square of the Baptistery of San Giovanni, seen from the threshold of the church of Santa Maria del Fiore in Florence. According to Manetti, Brunelleschi also invented an optical device called the Tavoletta that allowed anyone to physically experience how well the construction fitted in with the way of seeing the world. This device was essentially a small mirror held at arm's length and looked through a "lens-sized" hole in the panel. By moving the mirror in its plane, the correct alignment between representation and reality could be ascertained at the border of the mirror.

Brunelleschi-Alberti's method to represent space enabled the artists of the Quattrocento to claim they offered the spectator a sensation of "immersion" similar to that of our direct experience of the world. Unfortunately, this sensation disappears as soon as we move our eye away from the view point decided by the painter. Rare talented artists, such as Leonardo da Vinci in his painting The Last Supper (1495-1498), managed to achieve polyscopic perspectives by subtly assembling several central perspectives within the same painting [3]. But the technical advances of the time did not allow these artists to reach an authentic nomadism of vision [4] that only current technologies (synthetic holograms [4], augmented reality [5], etc.) could achieve. In addition, artificial perspectives generally break free from an important parameter of Brunelleschi's experience: the maximum aperture of the human eye. The consequence, especially in short distance representations, is an amplification of the perspective effect on the borders of the painting, referred to as lateral deformation by Panofsky and others [3], which is foreign to our habits of perception. Thus, while the naturalism of Tuscan artists has followed the laws of light, it has not been much faithful to those of vision.

At the same time in the county of Flanders, Jan van Eyck (1390-1441) was going his own way. Probably more than any other at that time, he was faithful to the light, notably in the restitution of contrasts, shadows, and reflections [6]. His representations, however, do not seem to conform to the geometry of an image formed on the wall of a dark chamber, as in his famous Arnolfini Portrait, where the ceiling joists and floorboards do not meet at a single point but at two points almost vertical to each other (Figure 1A). And while several authors have tried to search for alternative vanishing point hypotheses in this painting — see for instance Elkins's summary [7] no consistency was found in the proposed arrangements. The consensual hypothesis $[3,7,8]$ is that van Eyck painted on sight, without any concern for the laws of perspective. 

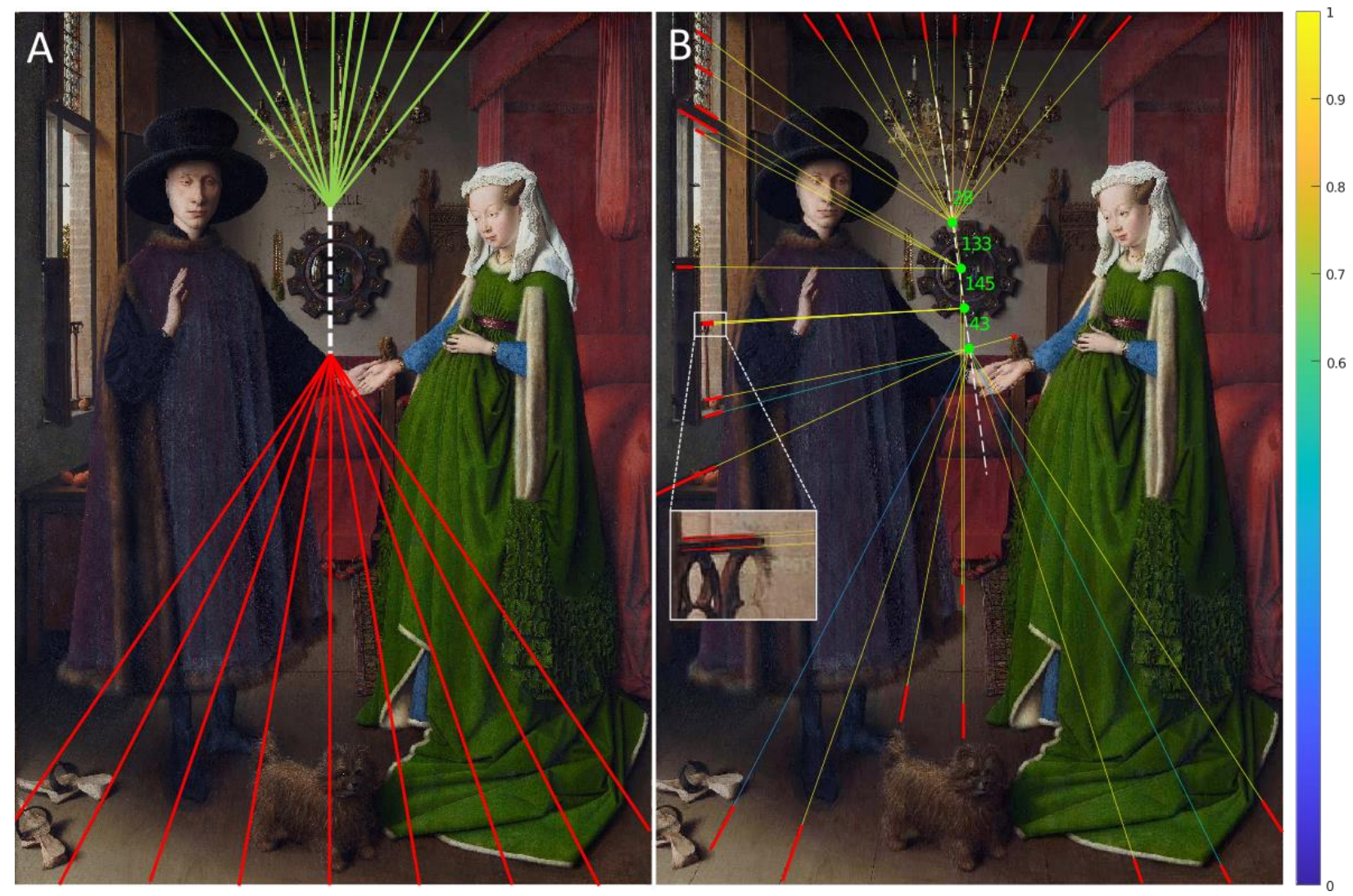

Fig. 1. Vanishing point reconstruction in Arnolfini Portrait, 1434, oil on oak panel, $82.2 \times 60 \mathrm{~cm}$, London National Gallery. A. Manual reconstruction. B. Reconstruction obtained with help of computer vision. (Background image [public domain], via Wikimedia Commons.)

\section{Method}

The coexistence of several vanishing point hypotheses for the same work is partly due to the fact that a theory or prejudice inevitably influences any perspective study. Karl Popper has shown that this is the case for any observational activity, and it is indeed easy to fit line segments into a preconceived scheme, allowing oneself "small twists" on the precision of their delineation. A few researchers in computer vision, such as Antonio Criminisi [8, 9], David G. Stork [8], and Andrew Zisserman [9], have shown that methods developed in this field can help to solve problems in art history. However, as far as the vanishing point problem is concerned, it must be admitted that a gap has grown wider between the way it is approached by computer vision scientists and art historians.

A graphic work certainly does not present with the same characteristics as photographs of urban scenes typically considered by vanishing point detection algorithms: Few edges are often exploitable, and their knowledge may be imprecise due to their delineation by the scholar and/or their representation by the painter. In this context, the expert's intuition remains of the utmost value, but computer vision can allow it to unfold with greater ease, precision, and confidence. Van Eyck's perspectives were thus analyzed using several tools that made it possible to: (i) measure the geometric consistency between a set of line segments and a vanishing point 
hypothesis, (ii) designate a group of segments and take into account the uncertainty of delineations to optimize their point of convergence, (iii) constrain the vanishing point, during this calculation, to remain on an established line, and (iv) automatically group segments specified in a painting and prove, using the a-contrario methodology, that the meeting points emanating from this grouping cannot result from chance.

The a-contrario methodology is inspired by the psychological theory of Gestalt, which considers that laws of grouping are at work in perception [10], with the fundamental principle that "we perceive immediately [mathematical transcription: our algorithm will detect] what cannot be due to chance." This principle was recently exploited in [11] to automatically detect vanishing points on the horizon line of a photograph. I adapted it to the specificity of a painting and to any number of horizon lines, as detailed in the supplementary material (SM). This set of tools constitutes a toolbox ${ }^{2}$, from which the most suitable tools can be chosen according to the context and one's thought process.

\section{Results}

Figure 1B shows the result obtained for Arnolfini Portrait: There are actually four centric points, roughly evenly distributed along an axis (called the axis of centers) that is slightly inclined. The geometrical consistency between the vanishing points and the delineating segments is indicated by the color of the vanishing lines, from dark blue for zero consistency to light yellow for a maximum consistency of 1: It is excellent everywhere, except for three lines on the ground. The fact that these lines are surrounded by perfectly consistent lines suggests an imprecision of their delineation rather than their not belonging to the group.

Figure 1B shows the values of -log10(NFA), where NFA represents the Number of False Alarms expected for a detected vanishing point (green points). The NFA expresses the number of times that an event as meaningful as the detected one is expected, on average, to occur by chance for a given number of line segments [10]. An NFA of less than 1 means in practice that an event cannot occur by chance. In fact, we obtain four NFAs much smaller than 1, which makes the existence of these vanishing points difficult to refute on a probabilistic level.

The other results are shown in Figure 2-the thought process followed to obtain some of them is detailed in the SM. Vanishing points in red did not emerge from the a-contrario analysis and therefore do not offer the same guarantee of existence as the others. They were, however, optimized in the sense of the maximum likelihood of the consistency criterion, and the consistency values finally obtained can be verified. It is noteworthy that the five paintings show a similar "fishbone" pattern [3], consisting of between two and four centric points aligned almost periodically along an inclined axis. This pattern has value of signature and may unravel attribution issues still in progress with some paintings (see the SM). Even more important for the

\footnotetext{
2 This toolbox has been coded in Matlab and built upon the $>\mathrm{V}<$ software associated with the method presented in [11] (https://members.loria.fr/GSimon/software/v/). I will work on making it available in a more user-friendly form than it currently is.
} 
history of art and science, this reveals that Jan van Eyck was making natural polyscopic perspectives at the same time that the Italians were inventing the artificial monoscopic perspective.

The horizontal vanishing point alignments in Saint Jerome in His Study (Figure 2A) and Madonna in the Church (Figure 2D) prove that van Eyck's perspectives were not geometrically constructed. Indeed, the only vanishing points that were allowed to be built during the first half of the fifteenth century were the centric point and the distance points, none of which were perceived as the projection of a 3D horizontal direction. On the other hand, the precision of the strokes and their consistency with respect to vanishing points leave little doubt as to the use of an optical device to represent space. This device had a glass window or flat mirror-the term glass is used in a generic sense in this paper-on which he drew the scene with a transferable carbon ink, by observing it from several viewpoints aligned along an inclined 3D axis, which is called the view axis in this paper. It is demonstrated in the SM that the view axis is similar, in mathematical sense, to the axis of centers.

The vanishing point reconstructions also reveal the existence of isolated centric points (Figures $2 \mathrm{~B}, 2 \mathrm{D})$, resulting from independent collages of monocular perspectives. The periodicity along the axis of centers is precisely broken in Madonna in the Church due to such a collage (outlined with dashed lines in Figure 2D).

\section{Van Eyck's Operating Procedure}

In order to better understand van Eyck's modus operandi, let us first note that the orthogonals of the analyzed paintings are invariably grouped inside horizontal strips. It can be deduced that van Eyck progressed his drawing strip by strip, from bottom to top or top to bottom. More specifically, the procedure used was as follows (in Figure 3, the painting progress is assumed from bottom to top, but a trivial inversion of terms describes a progression from top to bottom):

Initialization:

1. Put the eye at the lowest point of the view axis $(A, B)$.

2. Draw a horizontal strip at the bottom of the glass, delineating the space visible through it.

Repeat until the painting is complete:

3. Put the eye at the next point of the view axis (C-E, illustrating the last iteration).

4. Slide the glass vertically and horizontally to align the top boundary of the strip that has just been drawn with the real space observed through the glass $(F)$.

5. Draw a new strip in the extension of the last one by delineating the space observed through the glass $(\mathrm{G})$.

The alignment in stage four is very close to the one operable through the Tavoletta. However, it is more difficult to carry out because the change of view point between the drawing and the real view causes parallax effects that do not allow a perfect alignment at the boundary of the strip. A vertical edge, for example, is cut at the boundary into two edges still vertical but horizontally offset. This can be observed, e.g., in Arnolfini Portrait, though the effect is not perceptible at first 


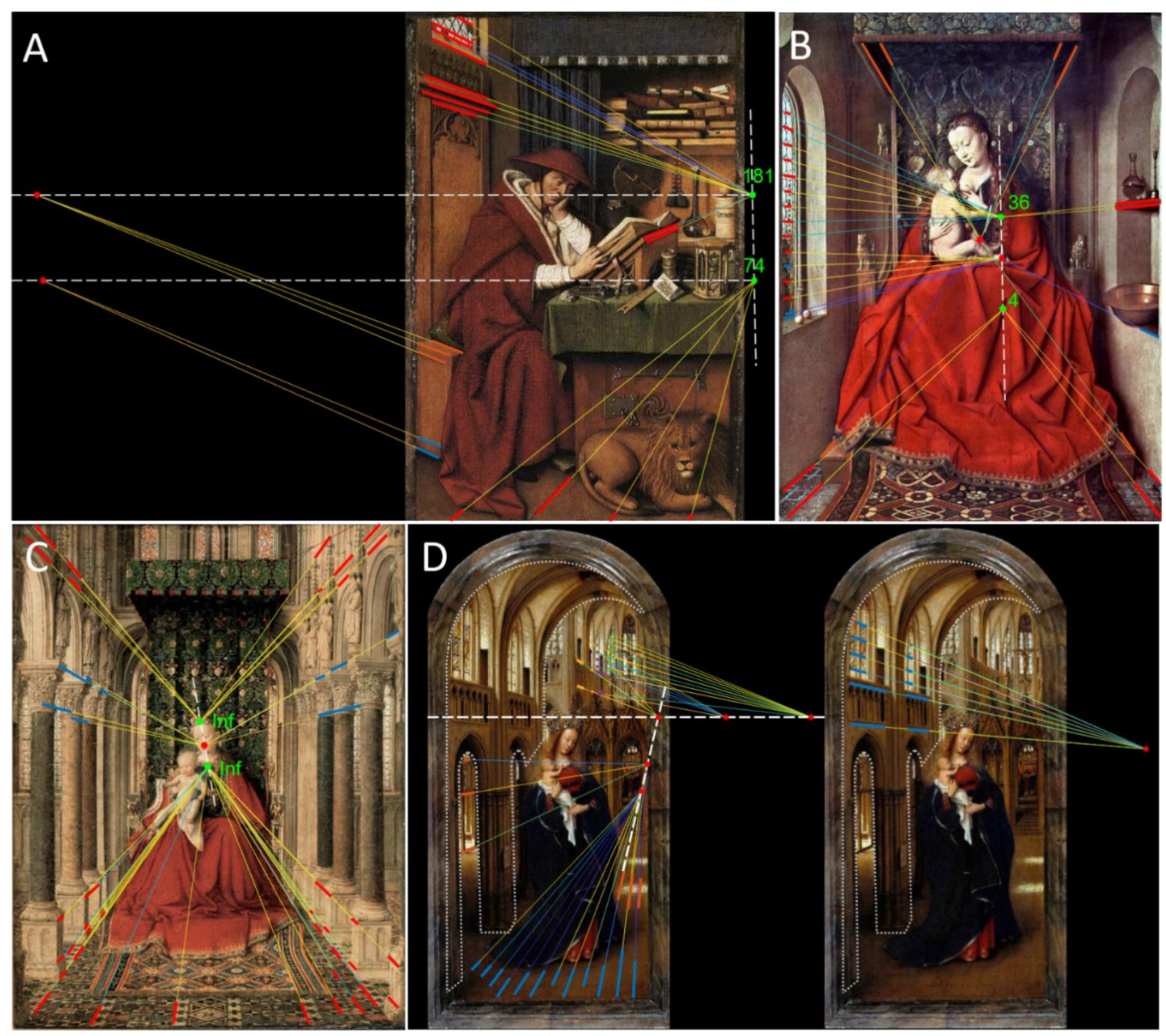

Fig. 2. Jan van Eyck's abstract signature in four others of his paintings. A. Saint Jerome in His Study, 1435(?), Detroit Institute of Art, $12.5 \times 19.9 \mathrm{~cm}$. B. Lucca Madonna, 1436, Städel Museum, $49.6 \times 65.5 \mathrm{~cm}$. C. Dresden Triptych, 1437, Gemäldegalerie, $27.5 \times 37.5 \mathrm{~cm}$. D. Madonna in the Church, 1437-1439, Gemäldegalerie, $14 \times 31 \mathrm{~cm}$. The graphic conventions are the same as in Figure 1. (Background images [public domain], via Wikimedia Commons.)

glance because it has been "smoothed" by van Eyck, resulting in continuous sloping lines instead of discontinuous vertical lines, which is obvious when the painting is flattened (Figures $4 \mathrm{~A}, 4 \mathrm{~B}$ ). It can also be observed on the chandelier, whose lower and upper parts belong to two different strips (Figure 4C).

Jan van Eyck could have sought to reduce "on average" the offsets along the strip boundaries, but he preferred to choose a vertical line across the painting, the leftmost one in the case of Arnolfini Portrait, as a reference registration axis common to all strips (one can see in Figure 4A that no offset appears along the leftmost vertical line, indicated in white). If van Eyck had not used this systematic method to deal with parallax, the centric points would not have been aligned in the paintings, which would have strongly compromised the discovery of the process. 


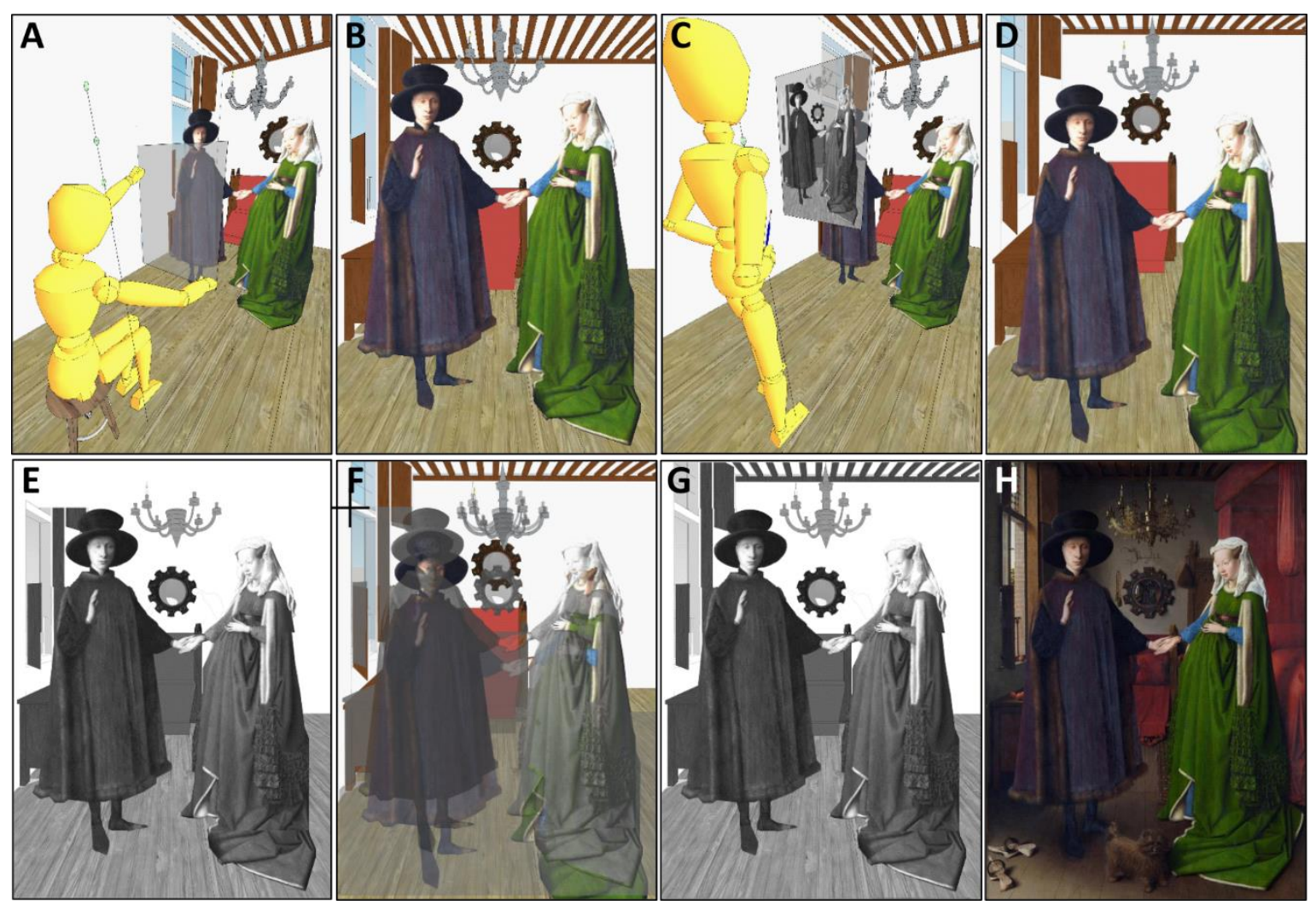

Fig. 3. Van Eyck's algorithm applied to the reconstructed model. The drawing is shown in black and white and the real views in color. A. Eye position and glass state (empty) at the beginning of the first iteration. B. View from the eye of the painter. C-D. Same figures corresponding to the beginning of the last iteration. E. Focus on the glass. F. Superimposition of the drawing (E) with the real view (D). The cross shows the assumed registration point. G. Result after drawing the last visible strip through the glass. $\mathrm{H}$. Painting. The complete simulation as well as a video animation are provided in the SM. (@2021, Université de Lorraine)

The viewing distance (distance between the view points and the glass) of Arnolfini Portrait can be calculated thanks to the presence of a round-tile stained glass window (see the SM). It is about $50 \mathrm{~cm}$, which allowed van Eyck to reach the corners of the painting with his brush without having to force the tension of the arm (Figure $3 \mathrm{~A}$ ). Knowing the focal length, the painting can be reconstructed in 3D by crossing the inverse rays passing through certain points of the painting with the incrementally built geometry of the scene (see the SM). The resulting 3D model is shown in Figure 4D. By applying the algorithm described above to this model, it can be verified that the simulated drawing (Figure $3 \mathrm{G}$ ) conforms to the real painting (Figure $3 \mathrm{H}$ ).

\section{Conclusions}

What could have led Jan van Eyck to follow such a complex drawing procedure? The 3D reconstruction of Arnolfini Portrait tells us that the heights of the eye along the view axis correspond to those of a man measuring $1.77 \mathrm{~m}$, beginning his drawing while sitting on a stool (Figure $3 \mathrm{~A}$ ) and ending it in a standing position (Figure $3 \mathrm{C}$ ). What is true for the painter is also true for the spectator, so that if you stand at a distance of about $50 \mathrm{~cm}$ from this painting, you will see Arnolfini's room in a centered view, whether you are small or tall, sitting or standing. But 

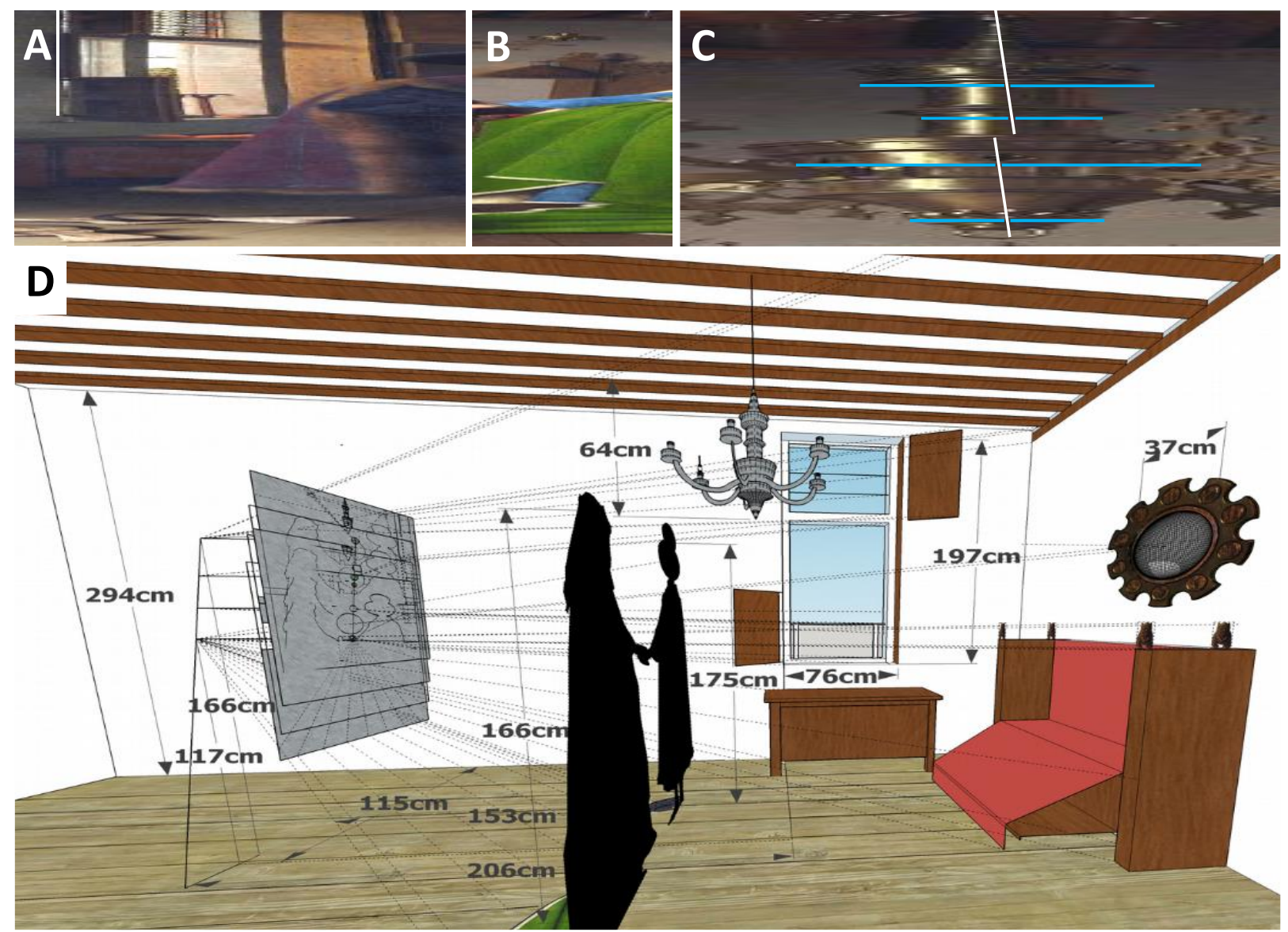

Fig. 4. 3D reconstruction of the view axis and Arnolfini's room. A-C. Parallax effects visible in the painting. D. 3D model obtained. The inverse rays used for the reconstruction are represented by dashed lines. (@2021, Université de Lorraine)

was van Eyck really interested in the question of the nomadism of vision sixty years before Leonardo da Vinci? This seems unlikely, all the more so since the physical centering of the isolated perspectives belonging to collages would require moving the eye to indeterminate locations. It seems more likely that he was preoccupied with lateral deformations or felt it was more natural to paint objects from the front. We are indeed used to seeing objects at eye level, i.e., from the front, rather than by raising or lowering our head. However, one cannot rule out the more prosaic hypothesis that van Eyck was forced to paint in several strips because of a narrow field of view, e.g., if he was looking at the scene through a hole as in Brunelleschi's experiment.

The inclination of the view axis is also somewhat puzzling. Was it unintentional (manufacturing defect, inclined floor)? Meticulous van Eyck would never have allowed such a default, visible to the naked eye (Figure 3A), especially since it significantly complicated the alignment of the strips. To paint Arnolfini Portrait, he imposed a horizontal distance of $66 \mathrm{~mm}$ between the lowest and the highest view points. This corresponds, within $1 \mathrm{~mm}$, to the average interpupillary distance of an adult man (see the SM). It is difficult to believe in chance under these conditions. However, the assumptions made about the vertical shifts are not applicable to the horizontal shifts because van Eyck chose to divide the drawing into horizontal strips. Would he have simply wanted to symbolically represent the stereoscopic human vision at the very heart of the drawing device? 
The study of the infrared reflectography of the drawing underlying Arnolfini Portrait may provide some clarification on these issues [12]. A change between drawing and painting on the ceiling seems to indicate that van Eyck was indeed concerned about lateral deformations (see the SM). Moreover, the superimposition of several representations of Giovanni-close to the glass, so that its perception from the left eye is noticeably different from that from the right eyeresembles an anaglyph and it is shown in the SM that it indeed corresponds to the superimposition of several views obtained from different heights of the view axis. The horizontal shift could therefore have been foreseen by van Eyck to allow him to choose between several appearances of objects according to aesthetic and symbolic criteria [12].

Finally, van Eyck represented space the way we see it-usually-and how he wanted it to be seen-through his collages and aesthetic or symbolic choices. By making a device to help him in this quest, he invented the natural polyscopic perspective. A future question might be how this affected his aesthetics, and we might reconsider our understanding of artistic choice versus technique. The similarity between Brunelleschi's device and that of van Eyck also raises questions and could well bring back into focus the hypothesis of a trip by van Eyck to Florence before 1430 [13].

\section{Acknowledgments}

I warmly thank my life partner, Marie Carmen Romero de la Rosa, with whom I have shared my discoveries, joys, and doubts from day to day, and whose subtle thought has greatly nourished mine. I would also like to thank all the people who will recognize themselves for encouraging me to progress in this work and particularly Erwan Kerrien for his thorough proofreading of the manuscript.

\section{References}

[1] Leon Battista Alberti and Rocco Sinisgalli (Ed. and Transl.). 2011. On Painting: A New Translation and Critical Edition. Cambridge University Press, Cambridge, UK.

[2] Antonio di Tuccio Manetti, Howard Saalman, and Catherine Enggass (Transl.). 1970. The Life of Brunelleschi. Pennsylvania State University Press, University Park, PA.

[3] Erwin Panofsky. 1927. Die Perspektive «als symbolische Form.» Lectures of the Warburg Library, Leipzig/Berlin.

[4] Jacques Desbiens. 2009. The perspectives of synthetic holography. In Proceedings of the 8th International Symposium on Display Holography (ISDH 2009).

[5] Vladimir Geroimenko (Ed.). 2018. Augmented Reality Art: From an Emerging Technology to a Novel Creative Medium. Springer International Publishing.

[6] Maximiliaan Martens, Till-Holger Borchert, Jan Dumolyn, Johan De Smet, and Frederica Van Dam (Eds.). 2020. Van Eyck. Thames \& Hudson, London, UK.

[7] James Elkins. 1991. On the Arnolfini Portrait and the Lucca Madonna: did Jan van Eyck have a perspectival system? The Art Bulletin 73, 1 (March 1991), 53-62. 
[8] Antonio Criminisi and David G. Stork. 2004. Did the great masters use optical projections while painting? Perspective comparison of paintings and photographs of Renaissance chandeliers. In Proceedings of the 17th International Conference on Pattern Recognition (ICPR 2004), 645-648.

[9] Antonio Criminisi, lan Reid, and Andrew Zisserman. 2000. Single view metrology. International Journal of Computer Vision 40, 2, 123-148.

[10] Agnès Desolneux, Lionel Moisan, and Jean-Michel Morel. 2008. From Gestalt Theory to Image Analysis: A Probabilistic Approach. Springer-Verlag.

[11] Gilles Simon, Antoine Fond, and Marie-Odile Berger. 2018. A-contrario horizon-first vanishing point detection using second-order grouping laws. In Proceedings of the 15th European Conference on Computer Vision (ECCV 2018), September 8-14, 2018, Munich, Germany, 323-338.

[12] Rachel Billinge and Lorne Campbell. 1995. The infra-red reflectograms of Jan van Eyck's portrait of Giovanni(?) Arnolfini and his wife Giovanna Cenami(?). National Gallery Technical Bulletin 16.

[13] Penny Howell Jolly. 1998. Jan van Eyck's Italian pilgrimage: a miraculous Florentine Annunciation and the Ghent altarpiece. Zeitschrift für Kunstgeschichte 61, 369-394. 


\section{Supplementary Material}

Jan van Eyck's perspectival system elucidated through computer vision

Gilles Simon (researcher), Université de Lorraine, CNRS, Inria, LORIA, F-54000 Nancy, France. Email : gilles.simon (at) loria.fr.

This PDF file includes:

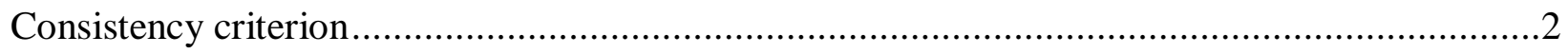

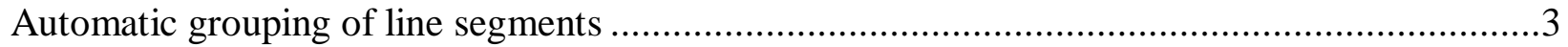



Perspective study of the Saint Jerome in his study .................................................................11

Perspective study of the Madonna in the church .......................................................................12

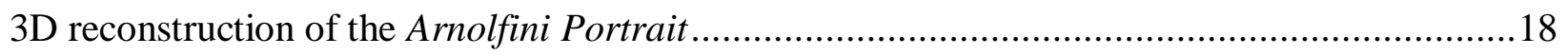

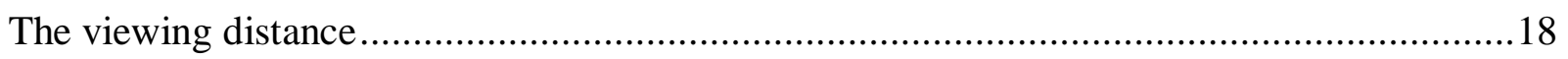



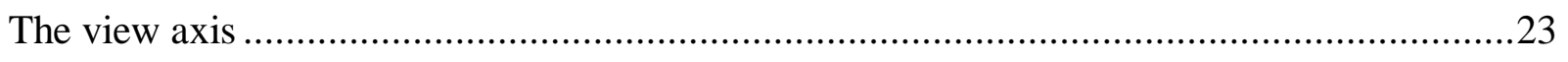





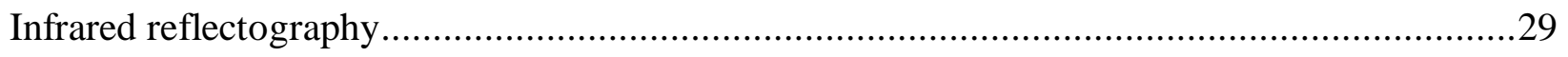



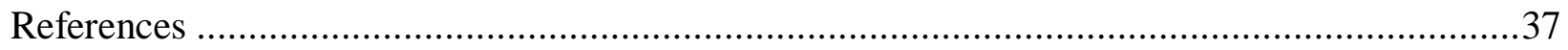

In order to distinguish between the references of the Supplementary Material and those of the main text (some of which are also cited in the Supplementary Material) the first are preceded by the letter $\mathrm{S}$, and the same for the figures. 


\section{Consistency criterion}

It is not so simple to design a criterion measuring the consistency between a line segment $\mathbf{s}$ and a point $\mathbf{p}$ supposed to be the vanishing point of s. It may seem natural to use the orthogonal distance between $\mathbf{p}$ and $\mathbf{s}$, and one of the first criteria was indeed the exponential of the opposite of this distance squared [S1]. But Y. Xu, S. Oh and A. Hoogs have shown that this measure is confusing because it gives maximum consistency when $\mathbf{p}$ is on (or close to) $\mathbf{s}$, either inside or outside s, while a finite segment of the image cannot contain its vanishing point [S2]. In fact, these authors have shown that most of the criteria proposed in the literature can be set to default in certain configurations. Their probabilistic criterion, on the other hand, considers the measurement error of the ends of the segments as well as their length, which is particularly suitable for the analysis of paintings:

$$
f_{c}(\mathbf{s}, \mathbf{p})=\frac{1}{\sqrt{2 \pi} \sigma} e^{-\frac{v^{2} L^{2}}{2 \sigma^{2}\left(u^{2}+(u-L)^{2}\right)}},
$$

where $L$ is the length of $\mathbf{s}$ and $(u, v)$ are the coordinates of $\mathbf{p}$ in a rectangular coordinate system centered at one vertex of $\mathbf{s}$ with $\mathbf{u}$-axis aligned with $\mathbf{s}$.

This criterion can also be used to, knowing a group of segments $\boldsymbol{s}_{i}$ supposed to meet at the same point, calculate the coordinates $\mathbf{p}^{*}$ of the optimal point in the sense of the maximum likelihood:

$$
\mathbf{p}^{*}=\arg \min _{\mathbf{p}} \sum_{\mathbf{s}_{i}}-\log \left(f_{c}\left(\mathbf{s}_{i}, \mathbf{p}\right)\right)
$$

The function minimized in (2) is not strictly convex, but $\mathrm{Xu}, \mathrm{Oh}$ and Hoogs found that it is almost always quasi-convex (unimodal) in a neighborhood of $\mathbf{p}^{*}$, so that any local optimum solver can find the global solution efficiently. 


\section{Automatic grouping of line segments}

The method used to automatically cluster a set of line segments into groups of meeting lines is inspired by the method proposed by G. Simon, A. Fond and M.-O. Berger to automatically detect horizontal vanishing points in photographs of man-made environments [11]. This method consisted of seven steps:

1. line segment detection based on the LSD algorithm [S3],

2. a-contrario detection of the zenith (vanishing point corresponding to the vertical direction of the scene),

3. a-contrario detection of horizon line hypotheses perpendicular to the zenith line (line joining the zenith to the centric point, assumed at the center of the photograph),

4. sampling (according to a Gaussian mixture model) of additional horizon line hypotheses around the previous ones,

5. a-contrario detection of vanishing point hypotheses along each horizon line hypothesis,

6. selection of the horizon line hypothesis obtaining the most consistent vanishing point hypotheses,

7. refinement of the vanishing point hypotheses on the selected horizon line using the expectation-maximization (EM) framework.

It is now described how each step has been adapted to paintings.

Step 1 (automatic segment detection)

Step 1 is replaced by manual designation of line segments for several reasons. First, I found that the segments detected with the LSD method were generally less accurate than those carefully delineated in enlarged views of the image. Second, segments of interest may not be detected by an automatic method because of their low contrast, and conversely, many useless segments may be detected. Finally, some segments are not defined by the edge of an object but by an alignment of objects. This is the case, for example, of the tops of ears of the two creatures embellishing the 
armrest of the sofa (Figure S1): the line segment connecting them is important because it represents the highest orthogonal, and therefore defines the boundary, of the lower strip.

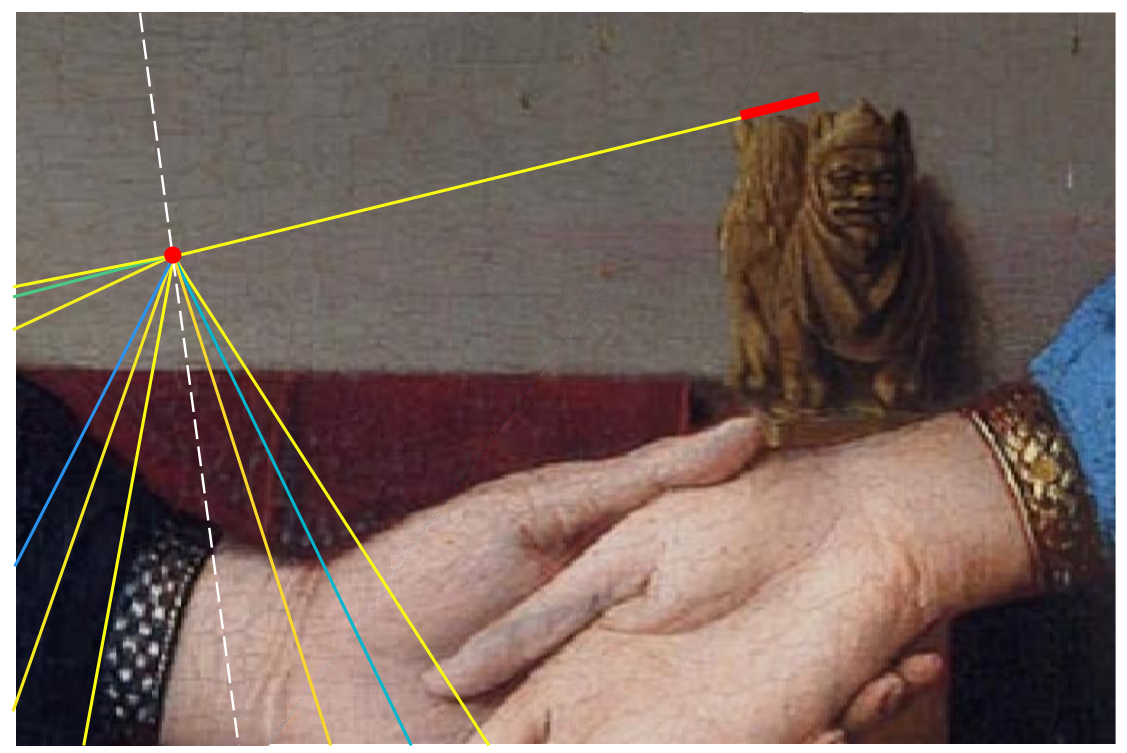

Figure S1. Some line segments are not defined by an edge but by an alignment of objects. Here the tops of ears of the two creatures define a line that passes through the bottom centric point of the Arnolfini Portrait.

Step 2 (detection of the zenith)

In artificial perspectives from the Renaissance to the present day, the zenith is generally located at infinity in the vertical direction in the painting. This is also the case in van Eyck's natural perspectives, e.g. the Arnolfini Portrait: in this painting, the horizontal line segments parallel to the plane of the back wall are also horizontal in the painting and the horizon line on the shutter passes through the centric point of its strip (see Section "Perspective study of the Arnolfini Portrait"): this only happens if the zenith is at infinity. If the glass was tilted down or up, the horizon line would be above or, respectively, below the centric point. The new method uses this hypothesis, in the sense that the candidate lines for the horizon line are taken horizontal in the image, though this hypothesis could be relaxed without difficulty. 
Steps 3 and 4 (a-contrario detection and sampling of horizon line hypotheses)

The a-contrario detection of horizon line hypotheses performed in [11] exploits the geometric property of 3D horizontal line segments at the height of the view point: these are projected onto the horizon line regardless of their direction in 3D. In photographs of man-made scenes, many objects are generally at eye level, resulting in an accumulation of line segments perpendicular to the zenith line on the horizon line. This makes it possible to detect the horizon line regardless of vanishing points. But this accumulation rarely occurs in paintings, i.e. in those of van Eyck, where only one line segment of this type could be found. For that reason, steps 3 and 4 were replaced by a basic sampling of $K$ horizontal lines linearly distributed between the top and bottom of the painting.

Step 5 (a-contrario detection of vanishing point hypotheses along the horizon line hypotheses)

The method in [11] adopted the following procedure: the line corresponding to the horizon line hypothesis under examination is cut into $M$ intervals more or less fine depending on the expected accuracy. For each interval, the number of (extended) line segments crossing the interval, and then the expected Number of False Alarms (NFA), i.e., the number of times, on average, that at least as many lines (among $N$ ) meet by chance within a similar interval, are counted. In order to compute the NFA, the background noise model of the event has to be known, i.e., in our case, the probability $p$ that a random line in the image domain crosses the interval by chance. This probability was calculated in [11] using integral geometry: its distribution function is constant inside the image domain, decreasing outside. Knowing it, we can evaluate the probability $p^{k}$ that $k$ lines of the image domain cross the interval by chance. But using this probability to detect vanishing points would cause a threshold issue. By contrast, counting the NFA according to the formula

$$
\mathrm{NFA}=M \sum_{j=k}^{N}\left(\begin{array}{l}
N \\
j
\end{array}\right) p^{j}(1-p)^{N-j}
$$

allows a more obvious threshold to be chosen: if the event under consideration is supposed to occur less than once by chance $(\mathrm{NFA}<1)$, it is considered meaningful. This reasoning can be extended to a multi-scale scheme [10] by calculating the NFA of all possible combinations of 
adjacent intervals, which implies replacing $M$ by $M(M+1) / 2$ in (3). The authors of [10] call Maximal Meaningful Mode a set of adjacent intervals, meaningful in the sense just mentioned and maximum in the sense of the inclusion of the intervals. The vanishing point hypotheses finally correspond to the peaks of the Maximum Meaningful Modes in the offset histogram of the meeting points between the horizon line hypothesis and all line segments. For better computational efficiency, the cumulative distribution function of the background noise is applied to the offset values before calculating the histogram, so that we obtain values between $-1 / 2$ and $1 / 2$ instead of $-\infty$ and $+\infty$ and equiprobable intervals with respect to the background noise.

This step is reproduced as is in the new method, except that a smooth counting of the number of line segments crossing an interval is performed. Indeed, fewer line segments are often available in a painting than in a photograph, and their measurement is more uncertain. This may result in intersection points located in different intervals for line segments supposed to converge to the same point, and a globally flat histogram from which no meaningful events is detected. To overcome this problem, a smooth counting is performed for each interval, by summing the consistency values from equation (1) between all the line segments $\boldsymbol{s}_{i}$ and the middle point $\mathbf{p}$ of the interval:

$$
k(\mathbf{p})=\sqrt{2 \pi} \sigma \sum_{\mathbf{s}_{i}} f_{c}\left(\mathbf{s}_{i}, \mathbf{p}\right)
$$

(the constant factor allows to add exactly 1 to $\mathrm{k}(\mathbf{p})$ where a line segment passes through $\mathbf{p})$. This counting is performed for the $M$ intervals of the $K$ horizon line hypotheses, leading to a $K \times M$ matrix of the values of $k$ obtained on a dot grid covering the whole image as well as its left and right extension to infinity (all results were obtained with $M=150$ and $K=M H / W$ for an image of height $H$ and width $W$ ). A visual representation of this matrix is interesting in itself to identify areas likely to contain vanishing points. The one obtained for the Arnolfini Portrait can be seen in Figure S2A. The defined segments are also shown (in red), so that one can find the segments involved in an area of vanishing points by going back to the sources of the "light rays" (high values of $k$ ) "illuminating" this area. These rays are curved outside the image (the left and right edges of the image are indicated by the white dashed lines), which comes from the application of the cumulative distribution function to the offset values of the meeting points as explained above. 


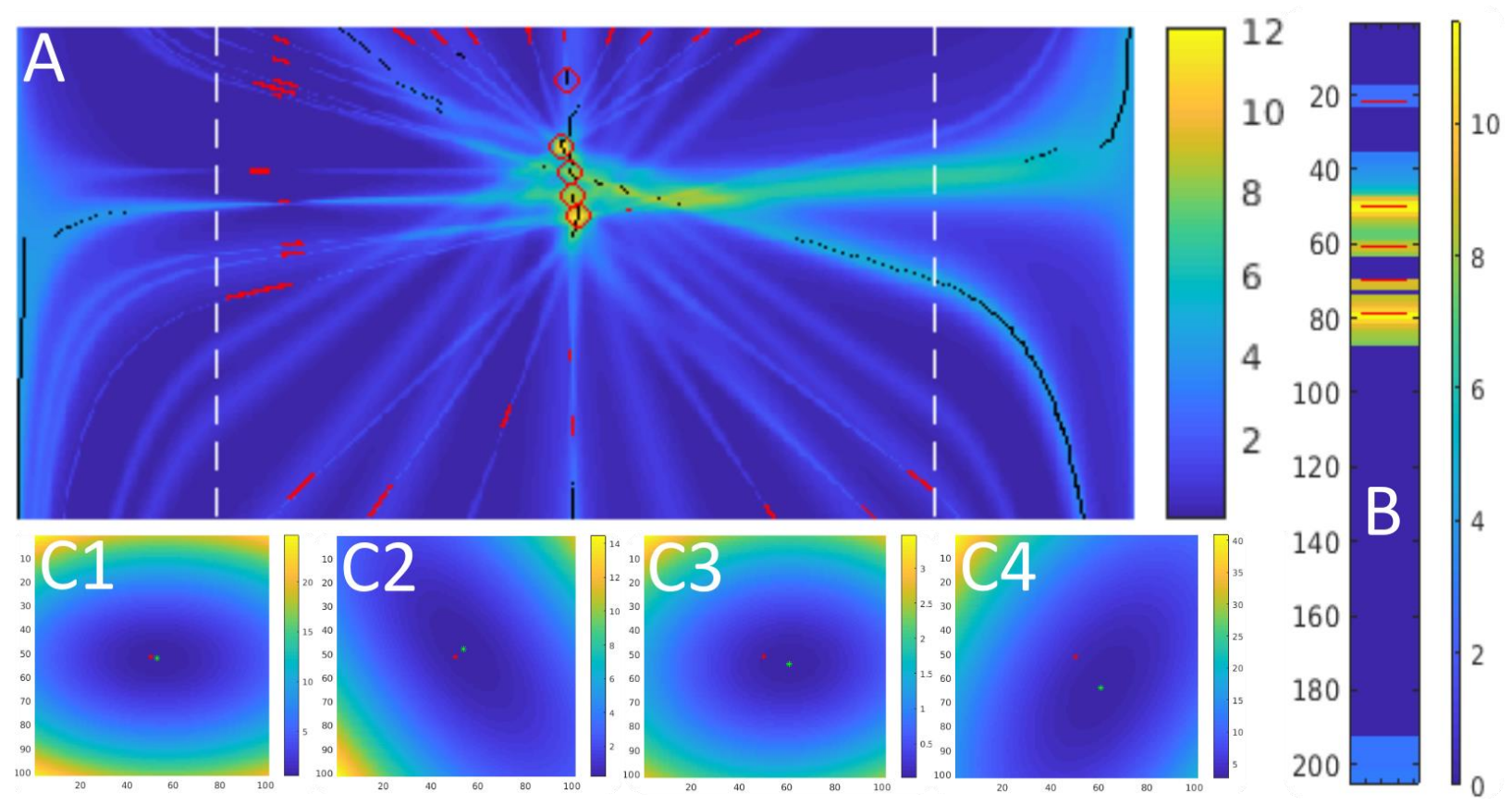

Figure S2. Automatic grouping of line segments and vanishing point refinement in the Arnolfini portrait. A. The point grid with $k$ values indicated by color mapping. The line segments are shown in red lines and the Maximal Meaningful Modes with black dots. B. Maximum consistency values over the grid lines filtered by a median filter of magnitude 3 . The local maxima are shown by red strokes and correspond to the centers of the red circles in A. C1-C4. Cost function and refinement result for the four vanishing points finally obtained.

The Maximal Meaningful Modes obtained on the grid are represented by black dots in Figure S2A. Several false positives are obtained here: it is indeed important to stress that this method is not a method for detecting vanishing points, but meeting points that cannot occur by chance. False positives are generally less meaningful than the true ones. They could be filtered out by lowering the threshold on the NFA or, more cleverly, by using a criterion such as the one proposed in [10] to limit the assignment of a segment to a single interval, i.e. the one whose NFA is the smallest among all those encountered by its supporting line. However, my goal was not to design a method to automatically detect vanishing points in a painting, but to help a scholar understand a perspective. In this sense, I preferred to keep all Maximal Meaningful meeting points rather than removing some of them at the risk of losing an effective vanishing point. On the other hand, the expert can designate an area of interest in which he concentrates his research. In that case, the entire grid is still considered for the calculation of the Maximal Meaningful Modes, so as not to corrupt the probabilistic criterion, but only the ones inside the area of interest 
are used in the next step of the algorithm. Thus, for the Arnolfini Portrait, I have delimited a vertical area of width a quarter of the image width centered in the middle of the image.

Step 6 (selection of the horizon line)

In [11], a score is first assigned to each vanishing point hypothesis by summing a consistency measure over all line segments, as in (4) though using a consistency criterion that does not consider the length of the segments nor their uncertainty. By contrast, the consistency criterion defined in (1), i.e. the score given by (4), already stored in the $K \times M$ matrix, is used with the new method. In [11], the horizon line hypotheses are scored by the sum of the two highest scores of the vanishing point hypotheses, or the score of the vanishing point hypothesis if there is only one, or 0 if there is none. Essentially looking for centric points and not wanting to take the risk of corrupting the score by false positives, I found more relevant, in the case of a painting, to take the maximum of the scores of the hypothesized vanishing points, or 0 if there is none. This value is computed inside the area of interest, but all the vanishing point hypotheses on the selected horizon line are considered in the rest of the algorithm, including those outside the area of interest. As an example, the scores obtained for the Arnolfini Portrait are displayed in Figure $\mathrm{S} 2 \mathrm{~B}$, from top to bottom respecting the position of the horizon line hypotheses. In [11], the horizon line hypothesis obtaining the best score is retained as the horizon line. In order to be able to detect several horizon lines in a painting, all local maxima of the score curve (red lines in Figure S2B and red circles in Figure S2A), previously filtered by a median filter of magnitude 3, are kept with the new method.

Step 7 (refinement of vanishing points)

The a-contrario analysis is able to prove, on a probabilistic level, the existence of a vanishing point. However, the EM refinement method can lead to vanishing points far from their initial position, in such a way that the evidence obtained for the initial point is no longer applicable to the end point. To tackle this issue, vanishing points are simply refined by maximizing the loglikelihood of the consistency criterion-Equation (2) integrating all the segments of the imageby restricting the search for the maximum to their neighborhood. Images $\mathrm{C} 1$ to $\mathrm{C} 4$ in Figure S2 show the values of the optimized function in the neighborhoods of the four Maximal Meaningful Modes finally obtained in the Arnolfini Portrait. The red dots represent the initial vanishing 
points, the green dots the refined ones. The function is locally convex for all four points. Finally, each line segment is assigned to the vanishing point with which it is most consistent. Vanishing points obtaining less than two segments are removed (e.g. the highest of the five in Figure S2A) and the segments possibly assigned to them are reassigned to the remaining ones according to the same criterion as previously. No threshold is imposed on the consistency of the segments, so that all of them are necessarily assigned to a vanishing point (if there is at least one) and it is up to the scholar to decide whether he wants to remove a line segment from a group because of its low consistency.

\section{Perspective study of the Arnolfini Portrait}

The Arnolfini Portrait (Figure S3A) is the painting whose in-depth examination led to the discovery of Jan van Eyck's drawing process. Its study was approached with the fishbone pattern dear to Panofsky in mind [3], characteristic according to him of the arrangement of vanishing lines in certain wall decorations of the 1st Pompeian century. Wondering whether this pattern could also describe the line arrangements in the Arnolfini Portrait, I looked for vanishing points along a presupposed axis of centers connecting the centric point of the ceil to that of the floor.

An encouraging sign came from the observation of the shutter in the middle left of the painting: its lower edge is clearly sloping, indicating that it is not seen from the front. But an alignment of nails on its upper reinforcement bar is perfectly horizontal in the painting (Figure S3B). This can only be coherent if the reinforcement bar was, in the space of the room, at the same height as the eye of van Eyck representing it, i.e. on his horizon line, the only locus where a line parallel to the horizontal plane but not to the image plane can be horizontal by perspective projection [11]. In addition, the three orthogonals at the top of the window appear to converge towards the point of intersection between this horizon line and the hypothetical axis of centers (Figure S3B). 




Figure S3. First signs suggesting a natural polyscopic perspective. A. The Arnolfini Portrait, 1434, Oil on oak panel, $82.2 \mathrm{~cm} \times 60 \mathrm{~cm}$, National Gallery. B. An alignment of nails on the upper reinforcement bar of the shutter is perfectly horizontal in the painting, and the three orthogonals from the window converge toward the meeting point between this alignment and a hypothetical axis of centers.

The four segments underlying the orthogonal lines of the window and the nail alignment were therefore added to the ceiling and floor line segments as input for the a-contrario analysis. It is important to note that the addition of the nail alignment, which does not represent an orthogonal of the scene, to the set of segments considered, is not prohibited: the a-contrario method does not detect vanishing points, but meeting points that cannot be due to chance. Moreover, any segment taken on the horizon line can take the role of an orthogonal, like that of any line parallel to the horizontal plane, because of the property stated above.

A first result was thus obtained, effectively showing three Maximal Meaningful Modes aligned along a slightly inclined axis. One problem remained: the horizontal bar of the balustrade was 
not consistent with any of the three centric points obtained. I therefore finely defined four segments on this bar (see the enlargement in Figure 1B of the main text) and added them to the set of segments already defined, as input for a new a-contrario analysis. The result is as shown in Figure 1B of the main text, with the orthogonal lines of the balustrade generating a new Maximal Meaningful Mode, with a particularly small NFA. This new centric point was precisely the one missing to obtain a near regular distribution along the axis of centers.

\section{Perspective study of the Saint Jerome in his study}

Since its acquisition in 1925 by the Detroit Institute of Arts, the Saint Jerome in his Study (Figure S4A) has been the subject of much controversy and its attribution to van Eyck is still discussed today [S4]. Some experts, such as Panofsky, claim that it was begun by van Eyck but completed by someone else, perhaps Petrus Christus (the date of 1442 inscribed in the painting is after van Eyck's death) [S5]. Others lean more toward a complete execution by a close successor of van Eyck who was familiar with the production and illuminations of his workshop. Maryan W. Ainsworth thus refers to "the awkward perspective of the chair [which] does not correspond to van Eyck's usual care in depicting a convincing, empirically conceived perspective" [S6].

The representation of the chair indeed looks awkward if one expects to see it in monocular perspective (Figure S4A). But the a-contrario analysis is irrefutable: it is a polyscopic perspective (Figure S4B). Two centric points, located towards the right border of the painting and defining a vertical axis slightly tilted, indeed emerge from this analysis. Their NFAs are much smaller than 1 and the consistency of the segments is generally very good, except in some places where the painter's strokes, or their delineation, are less precise. Most of the painting was done in a seated position: the horizon line of the upper centric point passes slightly below the eyes of Saint Jerome, himself seated. The lower strip of the painting, for which I see no other reason for being than to avoid too large a deformation of the floor, was painted in a crouching position. 



Figure S4. Perspective study of the Saint Jerome in his Study, 1432, Detroit Institute of Art. A.

The representation of the chair looks awkward if one expects to see it in central perspective. B. Vanishing point reconstruction.

Vanishing point corresponding to the three upper edges of the armrest and, respectively, the two lower edges of the chair, have been optimized under the constraint of remaining on the horizon line corresponding to the strip to which the edges belong. As a result, the consistency values are high and the line joining the vanishing points is near-parallel to the axis of centers. We can conclude that these two groups of edges correspond to the same direction of space and are perfectly coherent with a polyscopic_-here a stereoscopic_-perspective. This leaves little doubt as to the attribution of this painting - or at least of the strokes involved in the reconstruction of the vanishing points - to Jan van Eyck.

\section{Perspective study of the Madonna in the church}

Unlike the Saint Jerome in his Study, art historians today are generally in agreement about attributing the Madonna in the Church to Jan van Eyck. There is also little doubt that it is a mature work, made shortly before his death, around 1437-1441 [S7]. The impression of reality that emanates from this painting has prompted many researchers to try to identify the church depicted. However, it is now thought that it is not a building that existed: van Eyck is said to have combined elements from different churches [S7]. 




Figure S5. Perspective study of the Madonna in the church. A. Raw result of the a-contrario analysis applied to all edges likely to define a vanishing point. B. The parallax effect is perceptible on the interstitial lines of the floor crossing the first two strips. C. Enlargement of the floor area, showing an inaccurate geometry of the paving stones. D. Freezing of the vanishing point obtaining the lowest NFA and manual grouping of the other segments. E. Examination of the two-center perspective hypothesis. F. Detail. G. Examination of the three-center perspective hypothesis. H. Detail.

In fact, the reconstruction of the vanishing points in this painting has not been without difficulties. Figure S5A presents the raw result of the a-contrario analysis applied to all edges of interest: points resulting from accidental crossings lead to inconsistent groupings. On the other 
hand, the vanishing point obtaining the smallest NFA $(-\log$ NFA $=248)$ seems reliable: it is consistent with all the orthogonals of the triforium overhanging the nave, as well as with some orthogonals of the triforium overhanging the choir. I therefore first "freezed" this vanishing point and manually grouped together the other edges of the painting (Figure S5D): the last two stainedglass windows at the level of the triforium overhanging the choir do not contain orthogonals because they ensure the geometric transition between the orthogonal plane of the painting (including the first two windows) and the frontal plane including the stained-glass window visible behind Christ on the cross at the back of the church. A group of segments was therefore considered for each of these windows (violet and green edges in Figure S5D).

The floor of the church also shows many orthogonals at the interstices. Two of them are cut by the Madonna's robe and the last one on the right runs from the bottom to the middle of the painting. A close observation of these three lines reveals a slight deviation beyond the Madonna, increasing from left to right (Figure S5B). Seeing a probable parallax effect, I separated the ground orthogonals into two groups, one for the portions of lines in front of the Madonna and another for the portions of lines behind it. I then calculated a vanishing point per group of segments by optimizing the consistency criterion according to (2). The two groups of ground segments indeed give rise to two quite distant vanishing points, the upper one being not very far from the horizon line corresponding to the freezed vanishing point. A weak consistency of some lower segments can be noticed, but since the painting is very small $(31 \times 14 \mathrm{~cm})$ some of the floor strokes are not very precise (unless it is the actual geometry of the paving stones) so that it is sometimes difficult to define a common interstitial segment for three supposedly aligned tiles (Figure S5C). The other two groups of segments, associated with the stained-glass windows in the background, give rise to two even higher vanishing points in the picture.

In view of these results, I studied whether a perspective with two centric points (those of the ground, considering that the higher one is on the horizon line passing through the freezed vanishing point) could explain the whole set of segments (Figure S5E). This assumed that the freezed vanishing point is not a centric point, i.e., that its vanishing lines (colored blue) are not parallel to the interstitial lines of the pavement. This vanishing point being the most reliable (it emerges from the a-contrario analysis), it was assumed that the horizon line passes through it and the positions of the other vanishing points were constrained to remain on it during the 
optimizations defined by equation (2). The result can be seen in Figure S5E. We could already see in Figure S5A that some of the orthogonals of the choir are not consistent with the freezed vanishing point. We can also see that, assuming a two-center perspective, the (purple) segments associated with the third window of the choir are not consistent with the optimal point of convergence on the chosen horizon line (see enlargement in Figure S5F).

This led us to the following conclusion: either the triforium overhanging the nave is not parallel to the one overhanging the choir, which would be an aberration from an architectural point of view, or its representation is incoherent, from the perspective point of view, with the rest of the painting (in this case it would be a kind of collage).

In order to study whether the rest of the painting could be explained by a coherent polyscopic perspective, I then discarded all the line segments involved in the representation of the triforium of the nave, gathered together the orthogonals located at the level of the choir (orange segments in Figure S5G) and considered a single horizon line for the three groups of edges associated with the triforium of the choir. I had the choice of passing this line through one of the two vanishing points obtained in the previous step (purple and green segments in Figure S5D), these two points being at about the same height. I tested both options and kept the one that provided the best overall consistency. We can see, Figure S5G and in the enlargement Figure S5H, that the orthogonals of the choir (blue segments) are consistent with the optimized vanishing point on the selected horizon line, that the vanishing points of the third window (in violet) are much more consistent than in the hypothesis with two centric points (in particular the highest and lowest segments), as well as those of the fourth window (in green, see also the ends of the beam).

Finally, I still had to consider the three orthogonals located behind the arcade of the nave (brown segments). The lowest of them not being consistent with the freezed vanishing point, it was assumed that they were not part of the collage. They were grouped together with the three highest orthogonals of the floor and the position of the vanishing point of this group was optimized by forcing it to stay on the axis joining the other two centric points. The consistency of the orthogonal on the ground located behind the arcade is better in this configuration than in the two-center perspective. On the other hand, the highest orthogonal behind the arcade is less consistent: it "would like" to go up a little on the axis of centers, perhaps suggesting the 
existence of an additional centric point between the second and fourth ones, which we cannot verify because of the collage that occludes part of the observed church.

In conclusion, the three-center perspective, dissociated from the monoscopic perspective of the triforium of the nave, is more consistent than the two-center perspective supposed to explain the whole picture. Moreover, the collage hypothesis is confirmed if one accepts its provenance from a miniature of the missal of the Très Belles Heures de Notre-Dame entitled the Office of the Dead (Figure S6A). Albert Châtelet attributes this miniature to Jan van Eyck and proposes a dating around 1422-1425, when he was employed in The Hague in the service of the House of Bavaria [S8]. The association of red and blue in the keystones, as in the catafalque whose canopy is blue while the cloth covering the coffin is red, indeed evokes the coat of arms of this House, and the representation of certain characters at the bottom of the page shows similarities with characters from Jan van Eyck's Saint Barbara.

There is little doubt, geometrically speaking, that the representation of the triforium and the vaults of the nave is an enlargement of a portion of this miniature: same vanishing point, same curves in the ribs of the vaults with one exception (marked in yellow in Figure S6B). It is almost impossible to obtain two identical vanishing points from the same scene without one drawing being a copy of the other. In the case of a geometric construction, if a vanishing point is not in the center of the composition, its position is at the discretion of the painter. In the case of a painting from nature, to obtain the same vanishing point, one must position his eye at exactly the same place in the scene, orient the glass in the same way and place it at the same distance from the eye: this cannot be obtained by chance. Moreover, the perspective of the Office of the Dead has a defect, also reproduced in the Madonna in the Church: the alignment of the centers of the keystones (green line in Figures S6A and S6B) should be parallel to the orthogonals of the triforium, but it does not meet their vanishing point. Finally, the association of red and blue in the keystones, which was no longer imposed to van Eyck at the end of the 1430s (he working then in the service of the Duke of Burgundy) is also used in the Madonna in the Church. 

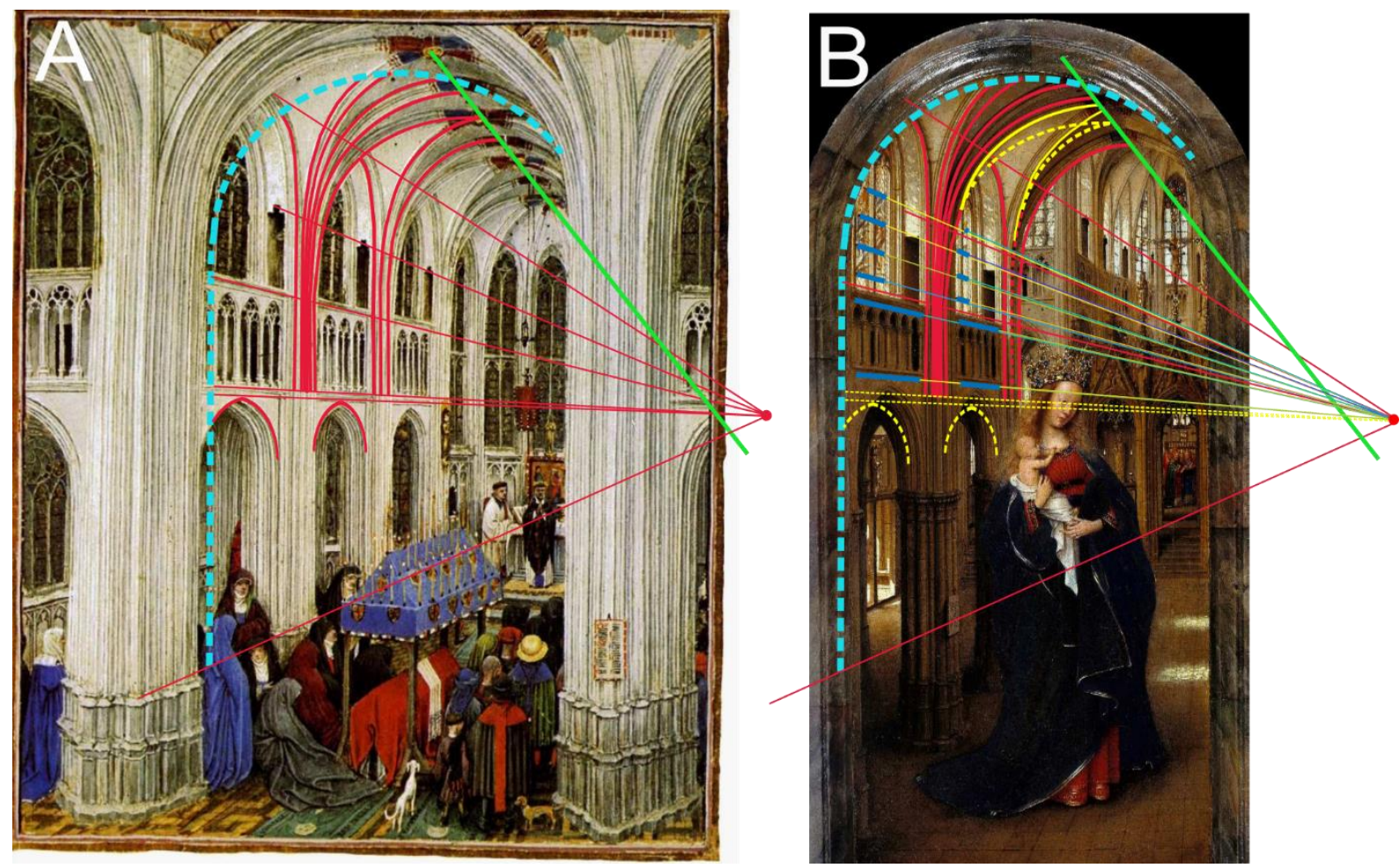

Figure S6. The collage detected in the Madonna in the church is very likely an enlargement of part of the Office of the Dead. A. The Office of the Dead, Hand G in the Turin-Milan Hours, Museo Civico, Turin MS 47. B. The vanishing point is exactly at the same position in the Madonna in the Church and most of the ribs of the vaults are perfectly aligned.

It is thus the polyscopic perspective with three centric points, completed by the monoscopic collage that better explains the geometry of the Madonna in the Church. This result also suggests that the church represented in the painting did indeed exist, since it was painted through a glass or mirror, and that it is therefore not vain to continue to seek to identify it, keeping in mind that only part of the painting is geometrically consistent with the interior of the church. Finally, the presence of a portion of the Office of the Dead in the Madonna in the Church is a further indication, but not proof, of the same hand at work in both paintings. 


\section{D reconstruction of the Arnolfini Portrait}

A 3D model of the Arnolfini's room was calculated in 2007 by researchers in computer graphics [S9]. Their reconstruction was based on several sources of measurements: the perspective of the painting, the rectified view of the image visible in the mirror and the assumed dimensions of some furniture of the time. But the accuracy of this model suffers from several approximations: a single centric point is considered in the painting, the image in the mirror is very small and the viewing distance was obtained by trial and error using a 3D software. By contrast, I took advantage of our new knowledge on the polyscopic nature of the perspective of this painting and the viewing distance was obtained by exploiting the diagonals of the round-tile stained glass window visible in it.

\section{The viewing distance}

It is known today that the direction $\mathbf{d}$ of parallel lines of space giving rise to a vanishing point is equal to the direction of the line joining the view point to the vanishing point. We can see this by understanding the vanishing point as the projection of the point located at infinity in the ddirection (Figure S7). The diagonals of the stained-glass window having an angle of $45^{\circ}$ with the orthogonals on the window, we get an angle of $45^{\circ}$ between the line joining the view point to the vanishing point and the line joining the view point to the centric point of the window's strip (Figure S7 illustrates this result by representing floor tiles instead of window tiles). From this it follows that the triangle formed by the view point, the centric point and the vanishing point is isosceles right-angled, i.e. the viewing distance is equal to the distance between the centric point and the vanishing point of the diagonals. It is important to stress that this reasoning could hardly have been adopted at the time of Brunelleschi and Alberti [S10]. The concept of infinite point indeed did not appear before the 17th century with Girard Desargues [S11], at the same time as the foundations of modern projective geometry. 


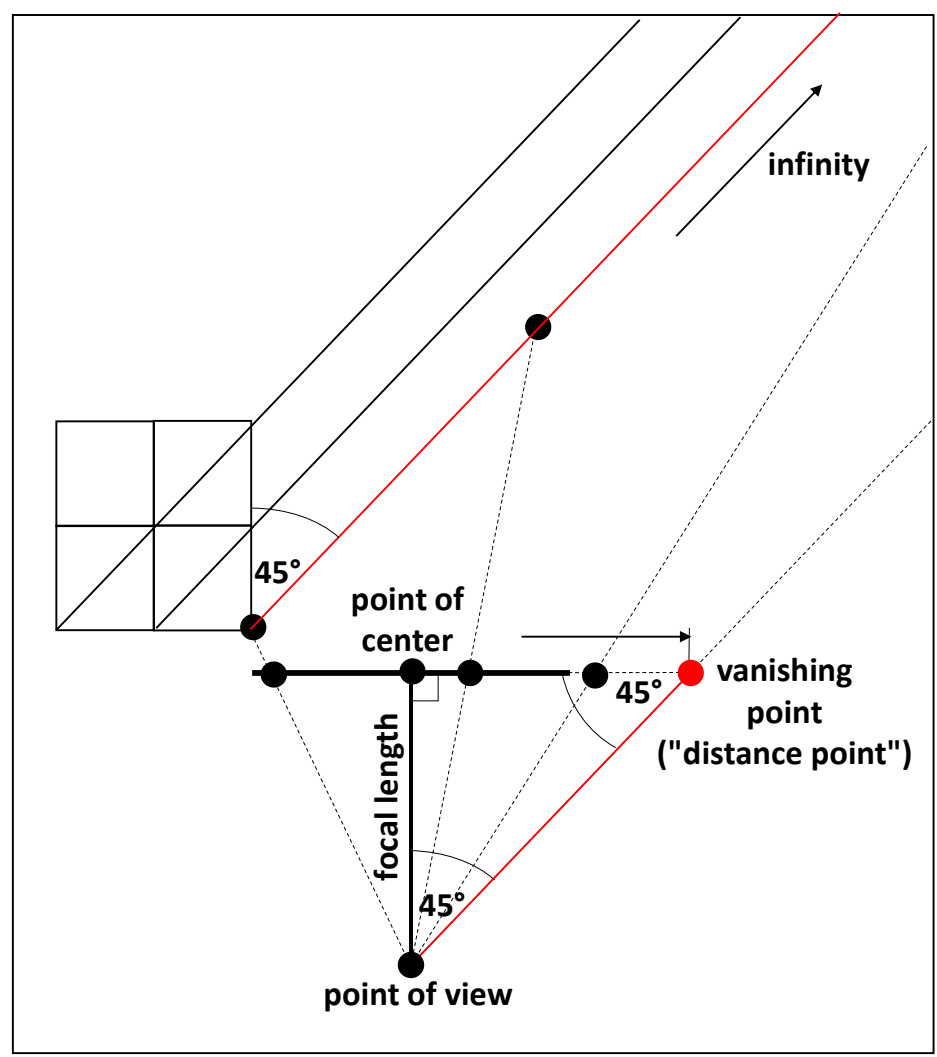

Figure S7. Geometry of a vanishing point seen as the central projection of a point at infinity (here in the direction of the diagonals of a square pavement). The scene is seen from the top.

The diagonals' vanishing point was obtained by maximizing the consistency of the line segments joining the tile centers (see enlargement in Figure S8A). As a vanishing point in a direction parallel to the left wall, it must also be on the projection of the line at infinity of that plane, i.e. the vertical line passing through the top centric point. This constraint was imposed during the optimization (Figure S8A). The consistency is very close to 1 for all the segments used. The viewing distance $f$ could therefore be measured with good accuracy in the painting and then converted into metric units, the aspect ratio of the painting being preserved in the digital image: we get

$$
f=49.7 \mathrm{~cm} .
$$



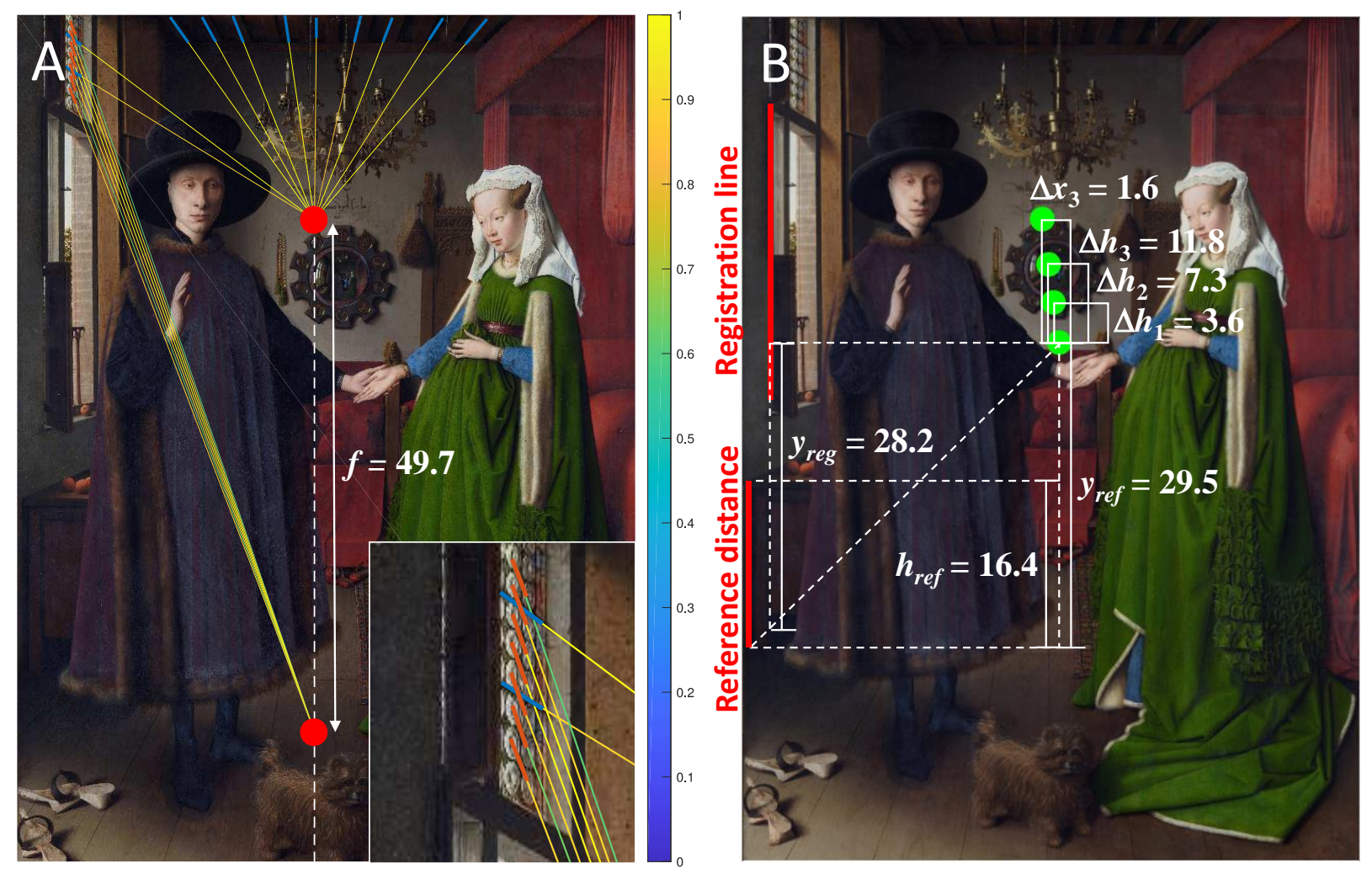

Figure S8. Useful measures for the reconstruction of the Arnolfini room and the view axis. A. Obtaining the viewing distance. B. Other measurements useful for reconstruction. All measurements are expressed in $\mathrm{cm}$.

\section{The room}

The glass (or the mirror) used by van Eyck to paint the Arnolfini Portrait was perfectly vertical and parallel to the back wall of the bedroom. Could it be that Jan van Eyck changed its distance to the eye or moved the whole device forward / backward during the operation? Let's see what this would imply. Each view point $i$ is assigned a 3D reference frame centered at $i$ whose $X, Y, Z$ axes are aligned with the axes of the room and oriented respectively to the right (looking at the scene), down and towards the scene. Let a 3D point of coordinates $(X, Y, Z)$ expressed in the reference frame 0 (assigned to the lowest view point). Its coordinates in the reference frame $i$ are equal to $\left(X-\Delta X_{i}, Y-\Delta Y_{i}, Z\right)$, where $\left(\Delta X_{i}, \Delta Y_{i}, 0\right)$ are the components of the translation to be applied to view point 0 to obtain view point $i$. According to Thales, we have (Figure S9A with $i=0$ ):

$$
x_{i}=\frac{f}{Z}\left(X-\Delta X_{i}\right), \quad y_{i}=\frac{f}{Z}\left(Y-\Delta Y_{i}\right)
$$





Figure S9. Perspective projection (A) in the case of a point of coordinate $(X, Y, Z)$ or a vertical segment of height $H$ whose base is on the ground; the view point is at height $H_{0}$ and $f$ represents the viewing distance (the scene is represented in side view), (B) in the case of a set of points located on a plane parallel to the image plane, at distance $Z_{\text {reg }}$ from the view point, when the latter evolves along an axis also parallel to the image plane. 
where $f$ is the viewing distance and $\left(x_{i}, y_{i}\right)$ are the coordinates of the point projected on the glass moved in $i$, expressed in the 2D frame of reference centered at the centric point $i$ and whose axes are aligned with the $X$ and $Y$ axes of the 3D reference frame.

Let us consider a set of coplanar points on a plane $Z=Z_{0}$. Equation (5) shows that they are transformed by a similarity of ratio $f / Z O$ between the plane and view $i$, and by a translation of coordinates $\left(-f \Delta X_{i} / Z_{0},-f \Delta Y_{i} / Z_{0}\right)$ between view 0 and view $i$ (Figure $\left.S 9 B\right)$. If the viewing distance $f$ or the depth $Z_{0}$ of any fronto-parallel plane had change between the views, the similarity ratio would have been directly impacted and all rectangles facing the glass would have changed in width between the strips. However, this is not what we observe in the painting, see for example Figures 4A and 4B from the main text: the vertical edges are oblique due to the smoothing of the parallax by the painter, but their apparent relative distance (for those in the same fronto-parallel plane) remains constant between the strips. We can therefore consider that the glass moves only in its plane and the axis of view remains fixed, parallel to the plane of the glass, throughout the whole operation.

In particular, the viewing distance calculated from the highest strip is also valid for the bottom strip. We can therefore reconstruct the portion of space visible inside the bottom strip. To do so, we start from an empty 3D map and introduce a point $C$ (the bottom view point), a bounded plane (the glass) at distance $f$ from $C$ and the $3 \mathrm{D}$ reference frame 0 . We also introduce a horizontal plane (the ground) at distance $H_{0}$ from $C$ downwards. To decide on the value of $H_{0}$, the height of the furniture under the window is used as reference measurement $\underline{H}_{r e f}$ : this value was estimated in [S9] equal to

$$
\underline{H}_{\text {ref }}=65 \mathrm{~cm} .
$$

Note that if this value was incorrect, our reconstruction would remain correct to within one scale factor. The apparent height of the piece of furniture $\left(h_{r e f}=16.4 \mathrm{~cm}\right)$ can be measured in the painting (Figure S8B), as well as the position of its base with respect to the centric point ( $y_{\text {ref }}=$ $29.5 \mathrm{~cm}$ ). According to Thales, we have (Figure S9A):

$$
\frac{H_{0}-H_{r e f}}{y_{r e f}-h_{r e f}}=\frac{Z}{f}=\frac{H_{0}}{y_{r e f}} \Rightarrow H_{0}=\frac{y_{r e f}}{h_{r e f}} H_{r e f} \text {, i.e. }
$$




$$
\underline{H_{0}}=116.9 \mathrm{~cm} .
$$

As the ground is now part of the 3D map, we can reconstruct about any point visible in the bottom horizontal strip, as long as it is connected to a point or plane already present in the map (initially, only the ground plane). More precisely, a point is reconstructed by crossing its inverse ray (discontinuous lines in Figure 4D of the main text) with a plane in the map or a line parallel to a frame axis passing through a point in the map. For example, the ground point of the vertical axis used by van Eyck as a reference for registering the strips is obtained by crossing the inverse ray of this point with the ground. Since its $2 \mathrm{D}$ coordinates can be measured in the painting $\left(y_{r e g}=\right.$ $28.2 \mathrm{~cm}$, see Figure S8B) and its $3 \mathrm{D}$ height $Y_{\text {reg }}$ is equal to $H_{0}$, its depth $Z_{\text {reg }}$ can be calculated by applying once again the Thales' theorem:

$$
\begin{gathered}
\frac{y_{r e g}}{f}=\frac{Y_{r e g}}{Z_{r e g}} \Rightarrow Z_{r e g}=f \frac{H_{0}}{y_{r e g}}=49.7 \times \frac{116.9}{28.2}, \text { i.e. } \\
\underline{Z_{r e g}}=206.0 \mathrm{~cm} .
\end{gathered}
$$

This procedure allows us to reconstruct the portion of space visible in the bottom strip (up to the segment joining the ears of the creatures). I assumed that the mirror was also drawn from this strip because reconstructing it from a higher view point would bring it too close to the sofa. I also assumed that the Arnolfini had been started and then completed from the lower view point and I simply back-projected them onto a fronto-parallel plane whose base corresponds to the inverse ray passing through Giovanni's foot. The other strips were reconstructed one after the other, from bottom to top, using the inverse rays from the other view points, after having reconstructed them according to the method described below.

\section{The view axis}

To reconstruct the view axis, no need to resort to new equations, we can simply observe Figure S9B. The plane parallel to the image plane passing through the registration line in $3 \mathrm{D}$ space (the depth of that plane is $Z_{\text {reg }}$ ) will be called the registration plane. Suppose that a point on the registration plane (e.g. the green point in Figure S9B) has been drawn on the glass from view point 0 and that the painter has translated the glass so that this point is aligned with its $3 \mathrm{D}$ 
counterpart by observing it from view point 1 . If other points on the registration plane have been drawn in view 0 (e.g. the blue point), they are translated in the same way and are therefore also aligned with their $3 \mathrm{D}$ counterparts by observing them from view 1, according to (5). This implies that we cannot know exactly which points on the registration plane (or, more probably, on the registration line) were used by van Eyck to register the strips. But what is most important to us is that when van Eyck was translating the glass from view 0 to view 1, he made the entire drawing undergo this translation, i.e. also the centric point corresponding to the bottom view (red point in view 0 Figure S9B). The latter can thus be considered in the new view (pink point in view 1) as the image of a virtual point located on the registration plane, corresponding to the orthogonal projection of view point 0 onto that plane. The same reasoning can be applied to any pair of views $i$ and $i+1$, so that we finally obtain that the viewpoint pattern is similar, in the sense of a similarity of ratio $Z_{0} / f$ to the pattern formed by the centric points. We can therefore calculate the relative positions of the view points by simply applying this similarity to the relative positions $\left(\Delta x_{i}, \Delta h_{i}\right)$ of the centric points, the values of which are shown in Figure S8B:

$$
\left(\Delta X_{i}, \Delta H_{i}\right)=\frac{Z_{r e g}}{f}\left(\Delta x_{i}, \Delta h_{i}\right) .
$$

The highest view point is thus at the height $H 4=H O+206.0 / 49.7 \times 11.8$, i.e.

$$
\underline{H 4=165.8 \mathrm{~cm}} .
$$

Anthropometric measurements from the DINBelg 2005 Project indicate that a man measuring $176.6 \mathrm{~cm}$ (the average height of Belgian men aged 18 to 65 in 2005) has, in a standing position, his eyes at $165 \mathrm{~cm}$ from the ground and, in a sitting position, a distance of $80.4 \mathrm{~cm}$ between his eyes and the flat of the seat $[\mathrm{S} 12]$. If the reference distance $\underline{H}_{\underline{r e f}}$ is correct, we can therefore assume that the height of the highest viewpoint corresponds to that of the eyes of van Eyck measuring $176.6 / 165 \times H 4=\underline{177.5 \mathrm{~cm}}$, and the lowest viewpoint to van Eyck sitting on a chair of $H O-80.4 \times 177.5 / 176.5=36.1 \mathrm{~cm}$ high. These posture hypotheses would remain valid in the case of an incorrect reference distance, provided that the size of van Eyck resulting from the adjustment of this distance remains plausible. The 5th percentile of adult Belgian male height is estimated at $164.1 \mathrm{~cm}$, the 95 th at 189.1 . The posture hypotheses therefore remain valid for $90 \%$ of men as long as the error on the height of the piece of furniture does not exceed $5 \mathrm{~cm}$. 
The horizontal distance between the lowest and the highest view point is equal to $\Delta X 4=206.0$ / $49.7 \times 1.6$, i.e.

$$
\underline{\Delta X 4=6.6 \mathrm{~cm}}
$$

Physiological measurements carried out in 1988 by the U.S. Army on men aged 17 to 51 years [S13] give a mean inter-pupillary distance of $64.7 \mathrm{~mm}$ with a standard deviation of $3.708 \mathrm{~mm}$. The value of $\Delta X 4$ can therefore correspond to van Eyck's inter-pupillary distance and this hypothesis remains valid for $90 \%$ of men if the error on the height of the piece of furniture is less than $7 \mathrm{~cm}$.

\section{The chandelier}

Since the chandelier has no point of contact, in the painting, with the other objects in the room, it was not possible to reconstruct it using the incremental reconstruction method described above. However, since its vertical axis (slightly inclined) crosses the two upper strips (as shown by the horizontal parallax in Figures S10A and 4C), it could be reconstructed by stereovision (Figure S10C - its inclination is assumed parallel to the image plane, which is an approximation). The body and the tube of the chandelier could then be reconstructed by back-projection, from their respective view points, of their silhouette onto the plane parallel to the image plane passing through the reconstructed axis. However, it is difficult to know from which view point, between the two upper ones, candle holders were drawn. We therefore examined the two hypotheses, and tried in each configuration to find the height of the plane perpendicular to the chandelier axis, optimizing the regularity of the angles between the lines joining the intersection of this plane with the chandelier axis to the centers of the candle holders (Figure S10D). This was done by trial and error in a modeling software and a quite satisfactory solution (six angles close to $60^{\circ}$ ) could be obtained assuming a representation from the lower of the two view points. A chandelier arm was finally cut out, with considerable simplification, from the most frontal view (considering that it belongs to the plane passing through the axis of the chandelier and parallel to the image plane) and duplicated five times with appropriate rotations.

Figures S10E and S10F show the 3D model obtained, reprojected according to the view points used for the reconstruction. Finally, figure S10G shows the union of the lower part and the upper 
part of the chandelier, projected according to their respective view points. The alignment between the model and the painting is relatively precise, although not as good (apart from simplification) at the arms. Antonio Criminisi and David G. Stork had already shown that the geometrical transformation (called homography) most favorable to the alignment of one arm to another did not allow a precise superposition of the two arms [8]. The arm duplicated in 3D could therefore not correspond precisely to all the others. In [8], the alignment error is attributed to imperfections in the painting process rather than in the shape of the manufactured chandelier. This conclusion was deduced from a comparison with more precise alignments that could be obtained in photographs of chandeliers of the time. The reflectography of the underlying drawing [13] also reveals a good penetration of infrared waves at the arms, which appear transparent over the background hatching, unlike the body, the tube and the candle holders, which appear more opaque (Figure S10B). It is therefore possible that van Eyck painted the arms directly on the panel, without the help of the glass. But it is also possible that he indicated them roughly on the glass (essentially the light contrasts, see below) with some of the pigments permeable to infrared waves, with a view to reworking them more finely on the panel. 


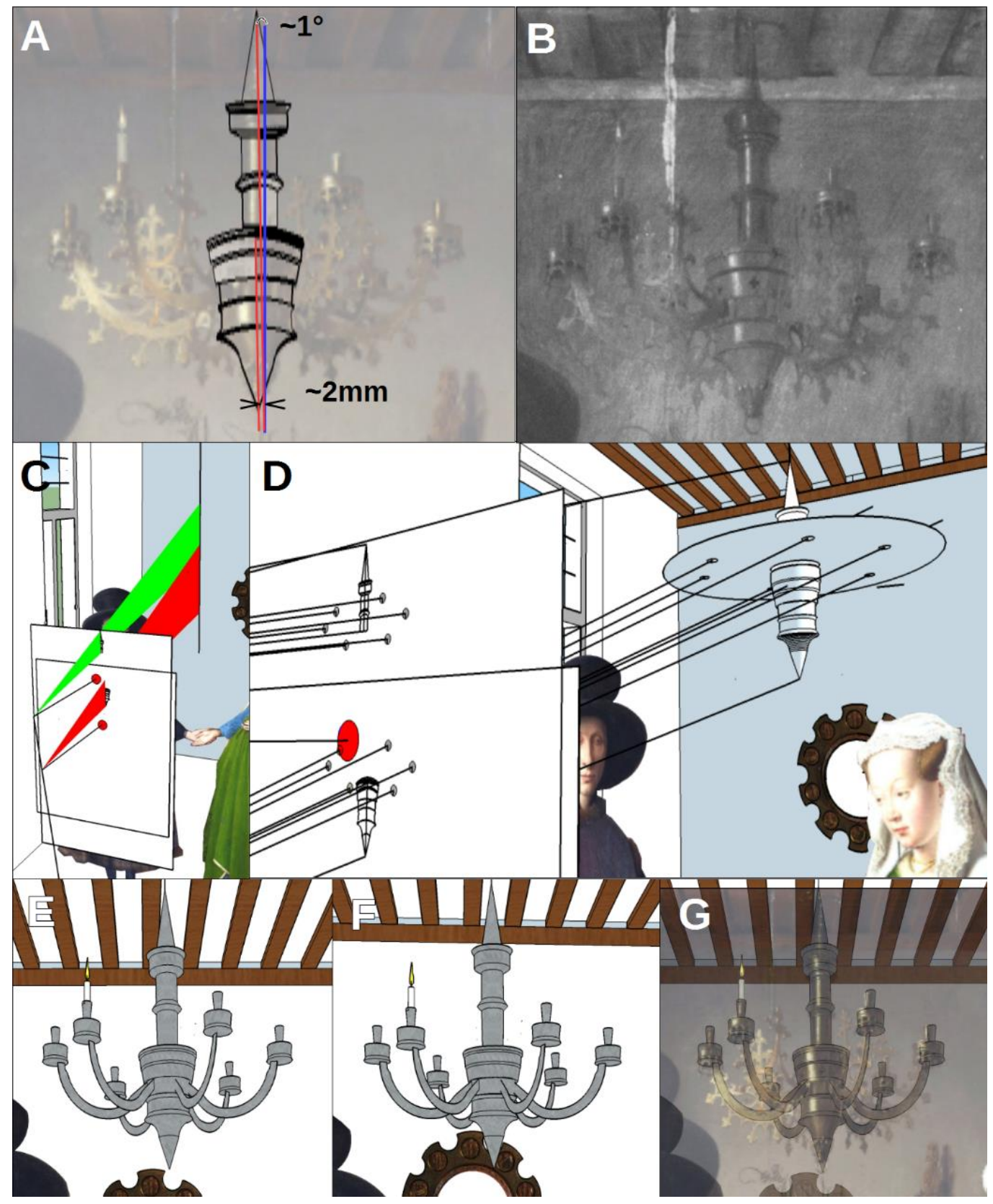

Figure S10. 3D reconstruction of the chandelier by stereovision. A. Highlighting the horizontal parallax and the inclination of the chandelier. B. Infrared reflectography of the painting in the area of the chandelier. C. Reconstruction of the axis of the chandelier. D. Reconstruction of the candle holders. E,F. Reprojection of the reconstructed chandelier in view 3 and (respectively) view 4. G. Union of the lower part of the chandelier reprojected in view 3 with its upper part reprojected in view 4 , shown transparently over the painting. 


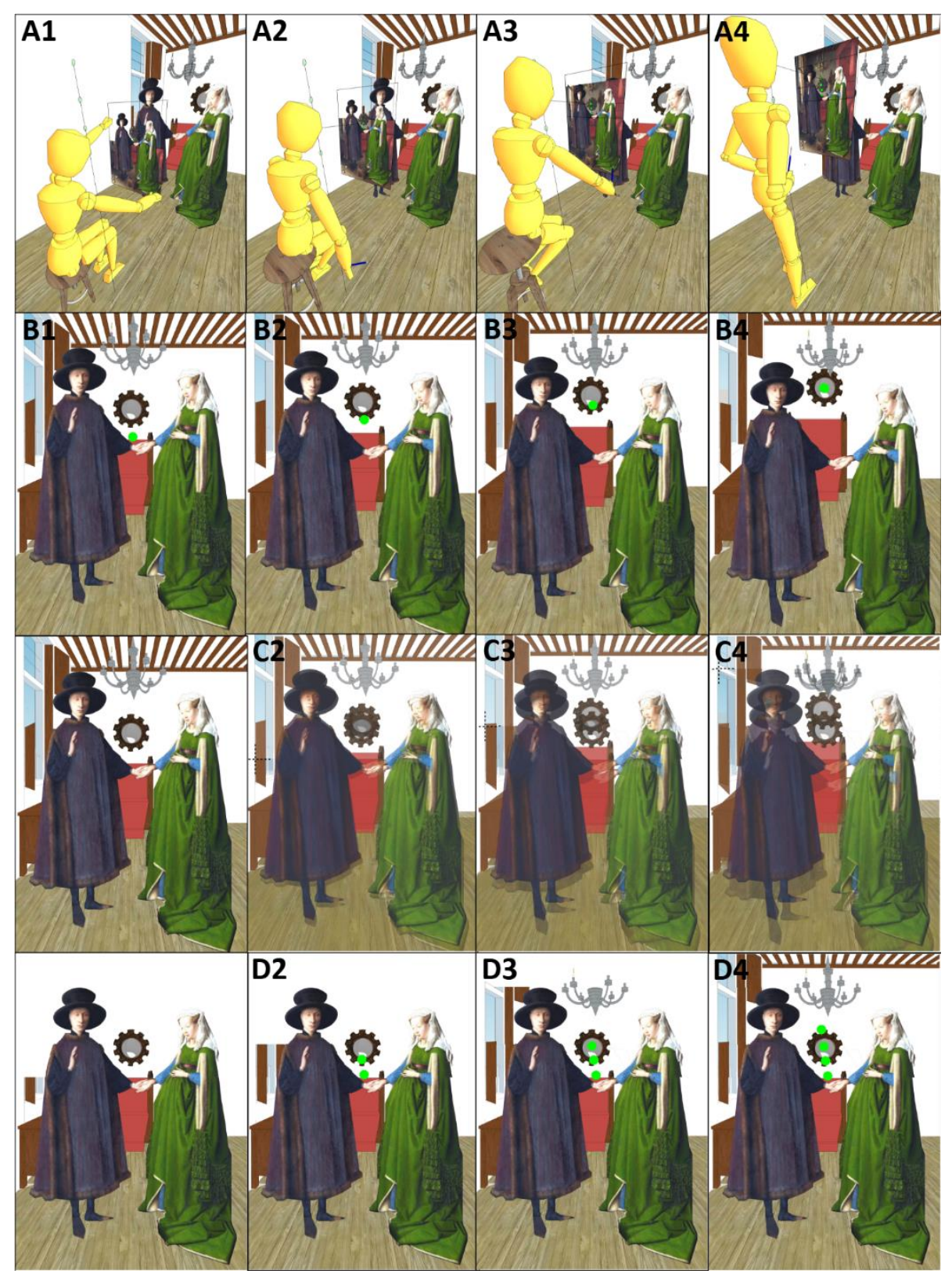

Figure S11. Simulation of van Eyck's drawing procedure on the reconstructed model. Col. 1. Initialization. Col. 2-4. Iterations. Row A. Positions of the painter's eye along the axis of view. The drawing on the glass corresponds to the one obtained at the end of each iteration. Row B. Views observed through the glass and corresponding centric points. Row C. Alignment of the drawing on the glass with the reality observed from the new view point. The assumed alignment point is indicated by a dashed cross. Row D. Drawing on the glass at the end of each iteration along with the cumulated centric points. 


\section{Full simulation}

Figure S11 and the video animation provided as part of the supplementary material, illustrate the full simulation of van Eyck's operating protocol, applied to the reconstructed geometry of the Arnolfini's room.

\section{Infrared reflectography}

The infrared reflectography of the drawing underlying the Arnolfini Portrait was taken and studied in 1995 by Rachell Billinge and Lorne Campbell for the National Gallery [13]. It shows an underdrawing that is, on the whole, faithful to the painting. This indicates that van Eyck scrupulously respected the progression of the drawing without overflowing the strips. One can even observe Figure S12A, above the sofa, a wide brushstroke that, extending horizontally to the right edge of the painting, seems to roughly delimit the lower strip. But the authors of [13] also noticed several changes between drawing and painting, mainly in the architecture and representation of Giovanni. In some cases, the painted part was also drawn, in addition to one or more variants which can be seen to correspond to the same objects represented from different positions on the view axis.

For example, a "dark shadow near the top of the window" seems to outline the contours of a lower ceiling, which the authors of [13] ultimately deemed unlikely because "it would have made Arnolfini unusually tall". This trace of a rising ceiling (and shutters) seems to indicate that the lateral deformations were indeed an integral part of van Eyck's naturalistic project. But the most spectacular changes are in the representation of Giovanni. See, for example, the superimpositions of drawings obtained at the level of his bust (Figure S12B) and lower body (Figure S12C): don't they look like anaglyphs? This seems anachronistic, but it is not: we obtain a similar result by superimposing several views simulated from different points on the view axis and aligned according to the procedure described above (Figure S12D, S12E). There are notable differences between photography and simulation, but it should be kept in mind that the virtual Giovanni has no thickness in the reconstructed model and the real Giovanni may have moved slightly between the execution of the strips. In any case it is indeed a parallax effect that is at work in these superimpositions, of the same nature as that predicted by the proposed model. 

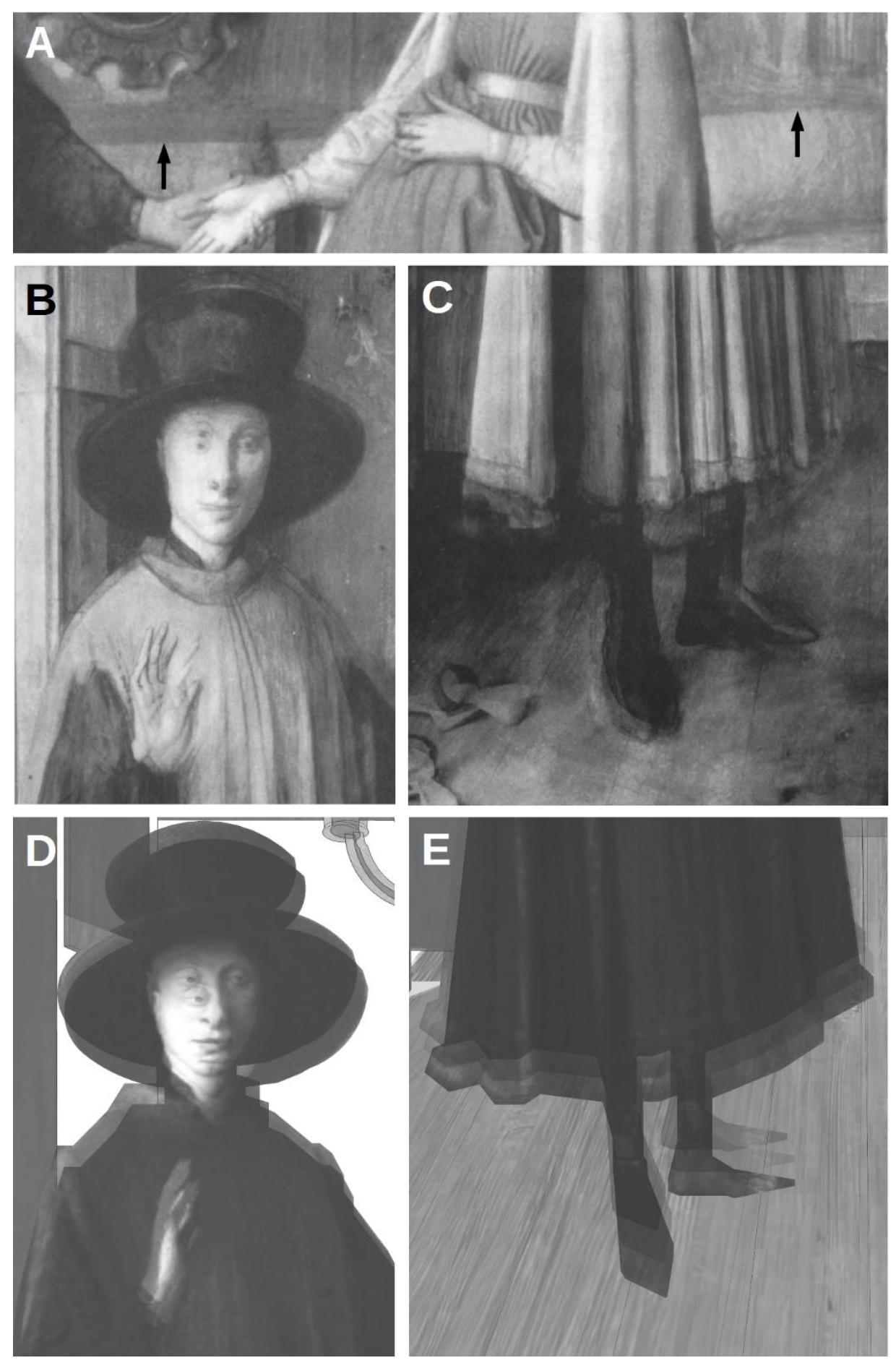

Figure S12. Underdrawing of the Arnolfini Portrait. A. A brushstroke appears to delineate two bands in the painting. B\&C. Details of infrared reflectography. D\&E. Overlay of the views computed from the first two (D) or three (E) view points. The infrared images were extracted from [13] CNational Gallery. 
The choices made by van Eyck in the representation of Giovanni are not a solution to the lateral deformations to which Giovanni is not exposed in the painting. Choices of a symbolic (Giovanni had to appear welcoming towards his witnesses) and aesthetic (the foot posture chosen was more graceful than the others) nature may be assumed [13]. This leads to another hypothesis concerning the inclination of the view axis: it would have allowed van Eyck to draw close objects from different angles in order to be able to choose, at the time of painting, the version best suited to his artistic intentions.

\section{Material and technique}

This section deals with the medium and material used by van Eyck for the realization of his underdrawings. It aims to show that the technical and cultural context of the beginning of the 15 th century allowed, if not favored, the emergence of the presumed technique.

Let us start with the support. It is obvious that van Eyck did not paint his polyscopic perspectives directly on the panel: the geometry of space is represented in a way that is far too precise (presence of parallax, etc.) to have been painted at a glance. The artist David Hockney raised a controversy in the early 2000s when he argued that van Eyck used a concave mirror to project, according to the principle of the darkroom, the image of objects directly onto the panel to be painted [S14]. He had certainly sensed that the geometry of certain objects-such as the Arnolfini's chandelier, which he rightly felt was seen from the front rather than from below [S15] — was too perfect to be painted without the help of an optical device. But Stork showed that the ambient light of an indoor space does not allow the assumed process to generate an image sharp enough to paint over the projected image: direct sunlight is required [S16].

In any case, it was not necessary to use such complicated devices to draw van Eyck's polyscopic perspectives. In his treatise on painting, Alberti suggests that a veil can be "very useful" to help with painting. He recommends placing it between the eye and the objects, "in such a way that the visual pyramid passes through the veil" [1]. The use of a veil seems to be excluded in van Eyck's case, because the texture of a veil does not allow for great precision and would be discernible in the underdrawing of the Arnolfini Portrait. But the presence of a convex mirror in the Arnolfini Portrait attests to van Eyck's interest in this object. 
It seems to me that observing the world through a flat mirror leads more naturally to (re)discovering perspective (assuming van Eyck was not informed of the Tuscan developments) than observing it through a window. For Alberti, the reflection in a mirror is the archetypal example of what the art of painting aspires to: “(...) if it is true that painting is the flower of all the arts, then Narcissus' fable is perfectly suited to painting. Is painting something else than the art of embracing the surface of a fountain in this way?" [1]. It is therefore quite possible that van Eyck alone rediscovered perspective, realizing that images formed naturally on a mirror (a flat surface) could be seen as a kind of painting with a strong power of illusion, which he, like many others, was looking for at that time [3]. This would have led him to delineate objects on a mirror and to discover, through experimentation, that this task could not be carried out without keeping one eye closed and the other in the same position throughout the execution of the drawing, i.e. drawing in central perspective.

According to Genevieve Warwick, "[a long] tradition of small, flat reflecting surfaces of polished metal, both circular and square, constituted the mirrors of the ancient world, as was known across the Renaissance. Steel gave a particularly clear and colourless reflection, and could be tempered into sheets of considerable size. These metal mirrors might also be silvered to increase the reflectivity of the surface finish, as was seemingly the case with Brunelleschi's panel in its reflection of the sky." [S17] Moreover, an examination of the Ghent Altarpiece reported by Panofski [S5] revealed that a sheet of silver constitutes the base of the layer of paint currently visible in the lower part of the central panel (that of the Lord) of the upper tryptic. Some authors also believe that Brunelleschi painted the Baptistery directly on a reflective material [S18]. If the hypothesis of van Eyck's trip to Italy is confirmed [14], it would therefore also be possible that this technique was passed on to him. Drawing on a mirror certainly presents difficulties, as the head, hand and brush obstruct part of the space to be represented. But sculptor and art historian Nigel Konstam has shown how to overcome these difficulties in practice [S19].

Discovering perspective by looking at space through a glass may be less natural. Alberti regarded perspective as "a window open to the world" [1] whose corners joined to the eye form a "visual pyramid". But, according to Gérard Simon, the very idea of a visual pyramid cannot emerge without a thorough knowledge of the optical works of Ibn al-Haytham (11th century), explaining vision by light rays entering the eye [S20]. Leonardo da Vinci drew in the codex 
Atlanticus a perspective machine with a glass and "a machine to fix your head in such a way that you cannot move it at all" (Figure S13). However, it must be borne in mind that he was benefiting at that time from the knowledge passed on by his predecessors, something we have no certainty about van Eyck. Moreover, transparent flat glasses were still difficult to manufacture at the time of van Eyck [S17].

The hypothesis of a glass, however, cannot be completely ruled out as painting on stained glass (without concern for perspective) was a common practice since the Middle Ages [S21]. Panofsky may also have given a clue in favor of a glass: he noticed that in the Madonna in the Church, sunlight enters from the north side, Gothic cathedrals being invariably oriented towards the east [S5]. Considering that it "would be almost sacrilegious to accuse [Jan van Eyck] of a fault against the simplest law of nature", Panofsky suggests that the laws of symbolism (light must arrive on the right side of the Madonna) have prevailed over the laws of astronomy. We now know that van Eyck relied on direct observation of the church to paint it, so it is very likely that the light itself was indicated on the glass (see below), and that it was by turning the glass over on the panel to transfer the drawing that the south became the north. If van Eyck had used a mirror, this operation would on the contrary have cancelled the symmetry already operated on the mirror. This clue, however, is most fragile.

Another important question is that of the medium. The infrared reflectography of the Arnolfini Portrait reveals that the drawing was executed in a liquid medium using a brush: "In some places, the fluid nature of the drawing material is particularly clear since pools of paint, which collect at the end of brushstrokes when a loaded brush is lifted from the surface, can be seen" [13]. It is also known that the magnetic waves used in reflectography have a frequency that allows them to be absorbed by the underlying carbon pigments, so that they appear as a negative in the photograph of the waves reflected from the background of the painting. It is therefore reasonable to assume that van Eyck painted his preparatory drawings with a liquid solution of carbon pigments. 


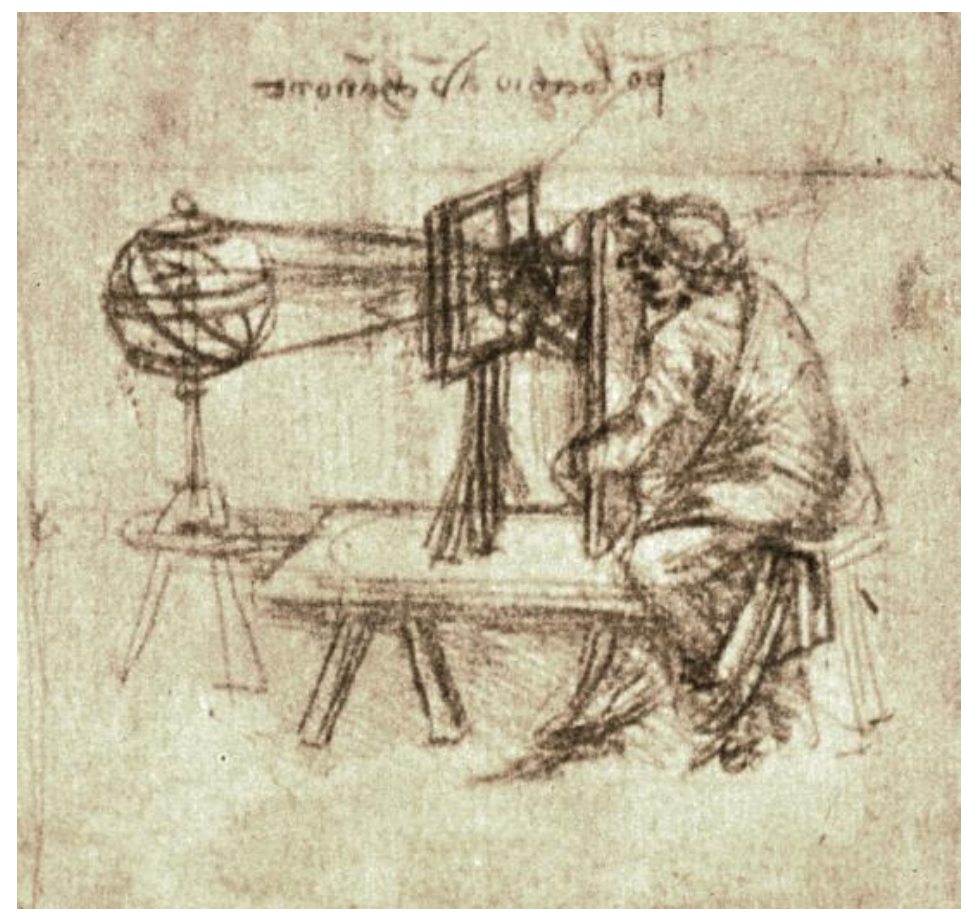

Figure S13. Leonardo da Vinci, Draughtsman using a Transparent Plane to Draw an Armillary Sphere, c.1510, Milan, Bibliotheca Ambrosiana, Codice Atlantico Ira (new 5). Drawing by Leonardo da Vinci illustrating the use of glass to draw in perspective: "Have a piece of glass as large as a half sheet of royal folio paper and set thus firmly in front of your eyes, that is, between your eye and the thing you want to draw. Then place yourself at a distance of $2 / 3$ of a braccia from the glass fixing your head with a machine in such a way that you cannot move it at all. Then shut or entirely cover one eye and with a brush or red chalk draw upon the glass that which you see beyond it; then trace it on paper from the glass, afterwards transfer it onto good paper, and paint it if you like, carefully attending to the aerial perspective."

The communicative power of carbon ink has been known for a long time. Giorgio Vasari concedes in his 1568 edition of the Lives of the Best Painters, Sculptors and Architects that Italian painting is indebted to Jan van Eyck's discovery of oil painting 150 years earlier. He also notes that the latter had a passion for experimentation, "like someone taking pleasure in alchemy" [S22]. Recent analytical techniques reveal that he used (but not invented) linseed oil, either raw or heat pre-polymerized, sometimes with the addition of a little pine resin [S23]. It is therefore quite plausible that he had also developed a medium helping carbon ink adherence and transfer. And it is also worth remembering that Gutenberg, a contemporary of van Eyck, shortly after his death perfected the water-based ink of copyists to obtain an ink suitable for printing, 
composed of resinous soot and linseed oil. If the idea did not come from van Eyck himself, it was at least in the spirit of the times.

Considering the above elements, the hypothesis that finally seems to me the most plausible, is that van Eyck made his underdrawings on a polished metal panel acting both as a mirror and as a material that can be painted and incised. This technique allowed him to draw accurate lines that he eventually combined with coarser brushwork and filled with ink afterwards. The authors of [13] point out that "The figures, the main architectural elements of the room and major pieces of furniture such as the bed are all outlined. Van Eyck has used a complex system of hatched brushstrokes to indicate areas of shadow. In flat areas such as the back wall and the floor between the figures, the strokes are roughly parallel and tend to slant down from right to left. In other areas such as the bolster and the bedspread the direction of the brushstrokes varies, giving a suggestion of volume, while the intensity of the overlapping lines indicates the depth of the shadows." Representing shadows and volumes by hatching will be, a few years later, a common technique in engraving (Figure S14, left). According to C. Gondard [S24], "In the field of engraving, Italy did not play the leading role it did in other disciplines, such as painting or sculpture: it was Northern Europe, Germanic or Flemish, that produced the best engravers. It is there, indeed, that the first authentic engravings were born." Figure S15, right, shows an example of a wood engraving made by a Master from Northern France around 1420-1430 [S24].

Finally, it is questionable whether certain areas of the Saint Barbara, a late work by Jan van Eyck (1437), have been obtained by this technique. For Panofsky, van Eyck considered this work as an autonomous drawing [S5], but more recent studies suggest that this interpretation denies the pictorial technique [S25]. Indeed, Till-Holger Borchert considers that if the landscape and the characters in the background are only sketched, the distinction between preparatory drawing and underlying painting is sometimes difficult to make. He thus discerns in the landscape and the site under construction "washes with Indian ink probably intended to define the effect of shadows during the pictorial act and the application of color glazes". It remains an open question whether these washes were transferred, and whether the finer strokes were the result of an engraving. It should also be noted that Borchert also finds, in several places, "highlights of white, generating a punctual accentuation of contrasts". This also raises the question of whether van Eyck also 
applied such highlights directly on the mirror, be it for this painting or any other, using pigments visible in the painting but undetectable, because non-carbon, to the infrared waves.


Figure S14. Left: M. Schongauer, Death of the Virgin, c.1471-1473, engraving, $17 \mathrm{~cm} \times 26 \mathrm{~cm}$, CBnF. Right: Master from Northern France, Madone, c.1420-1430, wood, $10 \mathrm{~cm} \mathrm{x} 13 \mathrm{~cm}$, CBnF. 


\section{References}

[S1] J. Kosecka, W. Zhang. 2002. Video Compass. Proceedings of ECCV, 47-490.

[S2] Y. Xu, S. Oh, A. Hoogs. 2013. A minimum error vanishing point detection approach for uncalibrated monocular images of man-made environments. Proceedings of CVPR, 1376-1383.

[S3] R. von Gioi, J. Jakubowicz, J. Morel, G. Randall. 2010. LSD: a fast line segment detector with a false detection control. Transactions on PAMI, 32, 722-732.

[S4] M. Faries. 2002. "Reshaping the field: the contribution of technical studies" in Early Netherlandish Painting at the Crossroads: A Critical Look at Current Methodologies, M. W. Ainsworth, Ed. Metropolitan Museum of Art/Yale University Press, 70-105.

[S5] E. Panofsky. 1953. Early Netherlandish Painting, Its Origins and Character. Harvard University Press.

[S6] M. W. Ainsworth.1994. "Workshop of Jan van Eyck" in Petrus Christus, Renaissance master of Bruges, M. W. Ainsworth, Ed. Metropolitan Museum of Art, 68-71.

[S7] S. Kemperdick. 2020. "Jan van Eyck's Madonna in a Church and its Artistic Legacy" in Van Eyck, M. Martens, T.-H. Borchert, J. Dumolyn, J. De Smet, F. Van Dam, Eds. Thames, 260-283.

[S8] A. Châtelet. 1993. Jean van Eyck enlumineur. Presses Universitaires de Strasbourg.

[S9] P. H. Jansen, Z. Ruttkay. 2007. The Arnolfini portrait in 3D — creating virtual world of a painting with inconsistent perspective. Proceedings of Eurographics, 25-32.

[S10] J. Green, P. S. Green. 1987. Alberti's Perspective: A Mathematical Comment. The Art Bulletin, 69, 641-645.

[S11] G. Desargues, R. Taton. 1988. L'œuvre mathématique de G. Desargues. J. Vrin.

[S12] DINBelg. 2005. "Body dimensions of the Belgian population". http://www.dinbelg.be/adultsmen.htm. 
[S13] N. A. Dodgson. 2004. Variation and extrema of human interpupillary distance.

Proceedings of SPIE, 36-46.

[S14] D. Hockney, C. M. Falco. 2002. Optical Insights into Renaissance Art. Optics and Photonics News, 11, 52-59.

[S15] D. Hockney. 2001. Secret knowledge: Rediscovering the lost techniques of the old masters. Viking Studio.

[S16] D. G. Stork. 2003. Color and illumination in the Hockney theory. Proceedings of the Color Image Conference, 11-15.

[S17] G. Warwick. 2016. Looking in the Mirror of Renaissance Art. Art History, 39, 254-281.

[S18] D. Gioseffi. 1957. Perspectiva artificialis. Per la storia della prospettiva, spigolature e appunti. Trieste, Tipografia Smolars.

[S19] N. Konstam. 2011. "Brunelleschi's First Experiments in Perspective-Nidel Konstam demonstrates". https://youtu.be/frB-XiXeNiQ.

[S20] G. Simon. 2001. Optics and Perspective: Ptolemy, Alhazen, Alberti. Revue d'Histoire des Sciences, 54, 325-350.

[S21] Theophilus Presbyter. 1963. On divers arts. University of Chigago press.

[S22] M. Gotlieb. 2002. The painter's secret: invention and rivalry from Vasari to Balzac. The Art Bulletin, 84, 469-490.

[S23] E. Effmann. 2006. Theories about the Eyckian painting medium from the late-eighteenth to the mid-twentieth centuries. Studies in Conservation, 7, 17-26.

[S24] C. Gondard. 2010. Histoire de la gravure occidentale : les origines. Bulletin de la Sabix, 47, 13-19.

[S25] T.-H. Borchert. 2001. «Sainte Barbe » in De Van Eyck à Dürer : les primitifs flamands \& l'Europe Centrale 1430-1530, T.-H. Borchert, Ed. Hazen, 145. 